\title{
The star cluster system of the NGC 7673 starburst ${ }^{\star}$
}

\author{
N. Homeier ${ }^{1,2}$, J. S. Gallagher III ${ }^{2}$, and A. Pasquali ${ }^{3}$ \\ 1 ESO, Karl Schwarzschild Str. 2, 85748 Garching bei München, Germany \\ 2 University of Wisconsin, Department of Astronomy, 475 N. Charter St., Madison, WI 53706, USA \\ 3 ESO ST-ECF, Karl Schwarzschild Str. 2, 85748 Garching bei München, Germany
}

Received 18 March 2002 / Accepted 7 June 2002

\begin{abstract}
We investigate the star cluster system in the starburst galaxy NGC 7673 using archival Hubble Space Telescope WFPC 2 broad-band images. For the first time we are able to examine the internal structures of the prominent optical clumps in this galaxy. The clumps are composed of young stars, 16-33\% of which are in bright star clusters. We identify 268 star cluster candidates in both the F555W and F814W images, and 50 clusters with the F255W filter. These data allow us to estimate ages and masses using color-magnitude and two-color diagrams for our sample. We find a population of young, $<6$ Myr clusters located throughout the galaxy with concentrations in the clumps. Star cluster mass estimates are $5-50 \times 10^{4} M_{\odot}$ for the brightest objects. The starburst remains active in physically well-separated regions, indicating a widespread starburst trigger. We discuss clump lifetimes, their implications for the future evolution of NGC 7673, and possible relationships to high redshift starbursts.
\end{abstract}

Key words. galaxies: evolution - galaxies: starburst - galaxies: individual: NGC 7673 - galaxies: interactions - galaxies: star clusters

\section{Introduction}

Starburst galaxies churn gas into stellar light at tremendous rates, so fast that the time to exhaust this material is short compared to the age of the universe (e.g., Mirabel 1992). In recent years they have also become notorious for their propensity to form swarms of star clusters, including massive, compact super star clusters (SSCs), which have been suggested by many authors to be the progenitors of globular clusters (e.g., Hunter et al. 1994; Whitmore \& Schweizer 1995; Ashman \& Zepf 2001).

The starburst phenomenon occurs rarely in the local universe, but increases in frequency at larger lookback times (e.g., Huchra 1977; Liu et al. 1998; Glazebrook et al. 1999 and references therein), which may be connected to the observed excess of distant "faint blue galaxies" (Ellis 1997). Studies of moderate redshift $(z \sim 1)$ galaxy samples reveal substantial populations of luminous galaxies with high star formation rates (SFRs) (Cowie et al. 1995; Omont et al. 2001). These include the blue "compact narrow emission line galaxies" (CNELGs; Koo et al. 1994; Koo et al. 1995; Guzmán et al. 1996, 1997, 1998; Phillips et al. 1997).

Similar objects have been categorized into various groups with various names, such as the "luminous blue compact galaxies", or LBCGs (our preferred term for such objects;

Send offprint requests to: N. Homeier, e-mail: nhomeier@eso.org

* Table 2 and Fig. 9 are only available in electronic form at http://www. edpsciences.org
Jangren et al. 2001). Galaxies of this class have small linear sizes $\left(<20 \mathrm{kpc}\right.$ ), luminosities near or above $L^{*}$, high surface brightnesses, strong emission lines, and blue colors, all features that are indicative of enhanced SFRs.

The evolutionary fate of moderate redshift LBCGs is a subject of some debate, and depends strongly on the current and future SFRs in comparison with the extent of their gas reservoirs. One scenario holds that the most compact of the LBCGs will evolve into spheroidal stellar systems covering a range in mass (e.g., Babul \& Rees 1992; Guzmán et al. 1998). The similarity of this subclass of LBCGs to luminous, young, starforming $\mathrm{H}_{\text {II }}$ galaxies has led to a classification of some systems as "H II-like" (Phillips et al. 1997; Guzmán et al. 1997).

Passive evolutionary models predict that after 4-6 Gyr, the luminosities and surface brightnesses of these $\mathrm{H}$ II-like LBCGs could resemble local low-mass ellipticals, like NGC 205 (Guzmán et al. 1998). The viability of this scenario depends on the star formation in LBCGs having a duration of less than $1 \mathrm{Gyr}$, and in that time they must lose almost all their gas (Pisano et al. 2001), which is difficult to accomplish in any but the least massive dwarfs (Mac Low \& Ferrara 1999; Ferrara \& Tolstoy 2000).

Alternatively, LBCGs could be analogs to intense starbursts in nearby disk galaxies, especially when observed in near faceon orientations that favor the escape of blue/ultraviolet light from the starburst region (Gallagher et al. 1989; Gallagher et al. 2000a; Barton \& van Zee 2001). The evolutionary scenario for disk-like LBCGs does not necessarily lead to their descendants 
being spheroidal galaxies. In nearby examples of this starburst mode, relatively minor interactions between galaxies can yield major starbursts in which the disk is only moderately perturbed (e.g., M 82). If the post-burst systems have sufficient gas supplies to allow star formation to continue, albeit at lower rates than in the current bursts, then they can appear at the present epoch as comparatively normal star-forming disk galaxies (Phillips et al. 1997; Hammer et al. 2000).

Related to these evolutionary questions is the issue of bulge formation. Theoretical work has shown that gas-rich $(>10 \%$ in $\mathrm{H}_{\mathrm{I}}$ ) disks subject to gravitational instability may form large clumps of gas, up to $10^{9} M_{\odot}$, which rotate in the plane of the disk (Elmegreen et al. 1993; Noguchi 1999). Such clumps experience strong star formation, resulting in a morphologically peculiar galaxy, and could appear like the "clumpy" galaxy investigated here.

The clumps suffer dynamical friction from the surrounding visible and dark matter, leading them to spiral inwards and accumulate in the central region, potentially forming a bulge. In this scenario the disk forms first, hosts the initial round of star formation, and produces a bulge. The remaining gas could then fuel a more sedate course of evolution. However, a key factor in this model is whether the clumps can retain their identities over the $\sim 100$ Myr time scales required for dynamical friction to act (Noguchi 1999).

To clarify the general issues of how starbursts connect to star formation processes and galaxy evolution, we concentrate on understanding nearby starbursts whose internal properties are observable. With this goal in mind, the Wide Field Planetary Camera 2 (WFPC2) Investigation Definition Team GTO program included exploration of small scale structures in blue starburst galaxies with $M_{B}<-18$ and high optical surface brightness, many of which are members of the LBCG class (Gallagher et al. 2000b). NGC 7673 (a.k.a. IV Zw 149, Markarian 325, UGC 12607, and UCM 2325+2318), a "disturbed spiral" or "clumpy irregular" galaxy was chosen for a multi-wavelength imaging investigation extending from the mid-ultraviolet to the near infrared. NGC 7673 is a member of the LBCG class, and a luminous infrared source (e.g., Gallagher et al. 1989; Hunter et al. 1989; Sanders \& Mirabel 1996). As such, it presents a unique opportunity to understand the inner workings of a high SFR object and its potential evolutionary connections to distant and present-day galaxy populations.

This paper presents an analysis of star clusters in the clumpy starburst regions of NGC 7673 based on archival images obtained with WFPC2 on the Hubble Space Telescope. We assume $H_{0}=70 \mathrm{~km} \mathrm{~s}^{-1} \mathrm{Mpc}^{-1}$; the recession velocity of $3408 \mathrm{~km} \mathrm{~s}^{-1}$ from the NASA/IPAC Extragalactic Database ${ }^{1}$ for NGC 7673 then implies a distance of $49 \mathrm{Mpc}$, and a projected scale of $250 \mathrm{pc}$ per arcsec. The distance modulus for this galaxy is 33.45 and one WFC pixel covers a projected scale of approximately 25 pc.

\footnotetext{
${ }^{1}$ The NASA/IPAC Extragalactic Database (NED) is operated by the Jet Propulsion Laboratory, California Institute of Technology, under contract with the National Aeronautics and Space Administration.
}

The next section summarizes properties of this unusual galaxy. Our observations and photometry of star clusters are detailed in Sect. 3, and Sect. 4 provides information on our choice of models for the spectral evolution of the clusters. In Sect. 5 we discuss the morphology of NGC 7673 as seen with WFPC2, Sect. 6 discusses the star cluster colors and magnitudes in terms of their ages, and Sect. 7 briefly characterizes the star-forming clumps. Our results are discussed Sect. 8, and in Sect. 9 we present our conclusions.

\section{Properties of NGC 7673}

The overall structure and kinematics of NGC 7673 were discussed by Homeier \& Gallagher (1999, hereafter HG), who argued that, despite the disturbed optical appearance of this system, it is a relatively unperturbed, rotating disk galaxy seen nearly face-on.

Initial investigations of NGC 7673 highlighted its disturbed and "clumpy" optical appearance (Markarian \& Lipoveteski 1971; Börngen \& Kalloglian 1975; Casini \& Heidmann 1976), and earned it a classification as a "clumpy, irregular" galaxy. The spiral pattern that is prominent in our images also was noted in earlier studies (de Vaucouleurs et al. 1976; Huchra 1977; Coupinot et al. 1982). Optical spectra revealed the presence of a broad emission component underlying the narrow lines more commonly associated with $\mathrm{H}_{\text {II }}$ regions, and a remarkably constant radial velocity across the galaxy (Duflot-Augarde \& Alloin 1982; Taniguchi \& Tamura 1987; Homeier \& Gallagher 1999).

H I observations of NGC 7673 and its companion NGC $7677\left(v_{\mathrm{r}}=3554 \mathrm{~km} \mathrm{~s}^{-1}\right)$ show that they have extended gas disks (Nordgren et al. 1997). NGC 7673 is apparently faceon and fairly regular, with a mild asymmetry on the western edge; NGC 7677 has a small outer extension pointing towards NGC 7673. The Hi mass of NGC 7673 is $4 \times 10^{9} M_{\odot}$, the dynamical mass (rather uncertain due to the low inclination) inferred from this line profile is $3 \times 10^{10} M_{\odot}$, with the resulting fractional Hi mass to total mass of $2 \%$ (Pisano et al. 2001).

The starburst activity is located within the inner half of the optical radius, concentrated in large complexes, the "distinctive clumps" in this galaxy (see Fig. 4). The outer disk is relatively smooth and nearly circular, but with ripples and wisps, likely remnants of a past interaction that could have triggered the starburst. The overall distribution of optical light, however, is highly asymmetric, with a rotational asymmetry value of $A=0.60$ (Conselice et al. 2000a). There is an optical shell $21 \mathrm{kpc}$ west of the main body (Dettmar et al. 1984; Homeier \& Gallagher 1999); such features most frequently are seen around spheroidal systems, but an optical shell is also present in the post-merger starburst galaxy NGC 3310 (Mulder \& van Driel 1996). NGC 7673 contains two strong non-thermal radio emitting regions characteristic of type II SN, indicating intense star formation over the last $\sim 3 \times 10^{7} \mathrm{yr}$ (Condon \& Yin 1990).

Due to its starburst, NGC 7673 is bright in wavebands that are sensitive to populations of young, massive stars, including the radio (Condon \& Yin 1990), far infrared (Klein et al. 1986; Hunter et al. 1989), optical emission lines 
(Gallagher et al. 1989; Guzmán et al. 1997), and ultraviolet (Benvenuti et al. 1982; Kinney et al. 1993). Its integrated SFR derived from the $\mathrm{H} \alpha$ and far infrared luminosities is 10 $20 M_{\odot} \mathrm{yr}^{-1}$ for a Kroupa (2002b) parameterization of the stellar initial mass function extending from 0.07 to $100 M_{\odot}$ (see Sect. 8.4). Selected parameters of NGC 7673 are summarized in Table 1.

\section{Data and observations}

The images analyzed in this paper were taken with the WFPC 22 and reduced via the standard pipeline. In Cycle 5, two $600 \mathrm{~s}$ exposures were taken with the F555W filter, and two $400 \mathrm{~s}$ exposures were taken with the F814W filter. During this set of observations, the main body of the galaxy was centered in the WF3 chip. In Cycle 6, three $800 \mathrm{~s}$ exposures were taken with the F255W filter. Again, the main body of the galaxy was centered in WF3, but with nearly a 90 degree offset in WFPC2 position angle from the previous observations.

The images were combined with the $\mathrm{IRAF}^{3}$ task IMCOMBINE, with the rejection method CRREJECT to remove cosmic rays. The images were divided by exposure time to yield final images in $\mathrm{DN} \mathrm{s}^{-1}$. In the remainder of this paper we focus our attention on an investigation of the rich array of compact star clusters revealed in the WFPC2 images.

\subsection{Cluster selection}

The IRAF packages DAOPHOT and APPHOT were used for the data analysis. We identified objects with the DAOFIND task in all three images. For the F555W and F814W images, we reran the photometry on each image using the coordinate list from the other image. We then matched the outputs and cleaned up any duplicates. From this list we matched the transformed F255W coordinates, resulting in 5 lists of objects: F255W, F555W, and F814W matched with each other, and F555W and F814W matched with each other. We kept objects with errors less than $0.3 \mathrm{mag}$ in the first set, and $0.15 \mathrm{mag}$ in the second set. We excluded objects fainter than 20.5 mag in F255W, and objects redder than 1.4 in [555-814] in the two filter list (none appear in the three filter list), which are most likely foreground stars. With this color criterion, we may be missing some dust obscured clusters, but without additional filters, we cannot distinguish between a dusty cluster and a foreground star. We also excluded 12 objects in the two filter sample farther than $16^{\prime \prime}$ $(\sim 4 \mathrm{kpc})$ from the nuclear region based on visual inspection. These objects were either obviously extended, associated with large background galaxies, or had colors and magnitudes inconsistent with clusters, even very old, very massive globulars. The two final sets contain 50 and 268 objects, respectively.

${ }^{2}$ Based on observations made with the NASA/ESA Hubble Space Telescope, obtained from the data archive at the Space Telescope Science Institute. STScI is operated by the Association of Universities for Research in Astronomy, Inc. under NASA contract NAS 5-26555.

${ }^{3}$ IRAF is provided by the courtesy of the National Optical Observatories, which are operated by the Association of the Universities for Research in Astronomy, Inc., under cooperative agreement with the US National Science Foundation.
While the data are sufficient to identify and perform photometry of clusters, they are not optimal for systematically examining sizes and internal structures. Due to this lack of spatial resolution ( 1 pixel covers $\sim 25 \mathrm{pc}$ ), our clusters are not necessarily tightly bound, and may range from relatively dense versions of $\mathrm{OB}$ associations to SSCs. Higher spatial resolution imaging would allow a refinement of this sample.

Aperture photometry was performed using the IRAF task APPHOT with a 2 pixel aperture radius. Background subtraction was done with an annulus of 5-10 pixels. Charge transfer efficiency (CTE) corrections were applied according to Heyer (2001). Gain corrections for all and aperture corrections for the F255W photometry only were applied following Holtzmann et al. (1995a,b, hereafter H95a/b). For the F555W filter, we determined aperture corrections to the standard 5 pixel aperture using three isolated, relatively bright objects available in the WF3 field. For the F814W filters, two of these same objects were bright enough for an accurate aperture correction measurement. We felt that as uncertain as this is, it is still preferable to using the H95a literature value. Aperture corrections determined in this way are -0.16 mag for F555W and -0.23 mag for F814W. The comparison to the literature values for the aperture corrections are -0.16 mag vs. -0.14 mag (H95a) for F555W, and -0.23 mag vs. $-0.18 \mathrm{mag}(\mathrm{H} 95 \mathrm{a})$ for F814W. There are no suitable objects in the $\mathrm{F} 255 \mathrm{~W}$ frame for determining aperture corrections, thus, we use the values found in H95a.

We utilize the WFPC2 synthetic magnitude system, with photometric zero points as listed in Table 9 of Holtzmann (1995b). The synthetic system was defined so that Vega magnitudes match $U B V R I$ measurements in the WFPC2 filter closest in wavelength to the $U B V R I$ filter. Thus the F555W and F814W magnitudes are very close to $V$ and $I$. We prefer to stay in the WFPC2 synthetic system for comparison to the models, as there is no satisfactory transformation from $\mathrm{F} 255 \mathrm{~W}$ magnitudes to $U$. We present a histogram of transformed $V$ and $I$ values for comparison to other studies in Sect. 7.

\subsection{Foreground extinction and reddening}

We have corrected our photometry for foreground Galactic reddening from Schlegel et al. (2000). At the position of NGC 7673 we adopt $A_{v}=0.124$ and $E(B-V)=0.04$. This amount of visual extinction translates into a neglible change of $-0.05 \mathrm{mag}$ in the [555-814] color, and $-0.13 \mathrm{mag}$ in [255-555].

\subsection{Completeness}

The completeness limit for our selection of nearly unresolved star clusters was determined for the F555W filter by adding 10000 artificial stars at each 0.5 mag step between 20 and 27 mag. The recovery fraction was calculated for each magnitude step at a range of background levels. At the highest background level in this galaxy $(500 \mathrm{DN})$, we have a $50 \%$ recovery rate at 26th magnitude in F555W. For 98\% completeness at this background level, the faint magnitude limit is 21.5 . 
Table 1. Parameters of NGC 7673.

\begin{tabular}{ccc}
\hline \hline Parameter & Value & Reference \\
\hline$\alpha, \delta(\mathrm{J} 2000)$ & $23: 27: 41.2,+23: 35: 21$ & Falco et al. (1999) \\
Redshift & $3408 \mathrm{~km} \mathrm{~s}^{-1}$ & Huchra et al. (1999) \\
Distance & $49 \mathrm{Mpc}$ & redshift and $H_{0}=70 \mathrm{~km} \mathrm{~s}^{-1} \mathrm{Mpc}^{-1}$ \\
HI Mass & $4.09 \pm 0.03 \times 10^{-9} M_{\odot}$ & Pisano et al. (2001) \\
$L_{\mathrm{H} \alpha}$ & $26.3 \times 10^{41} \mathrm{ergs} \mathrm{s}^{-1}$ & Gallego et al. (1997) \\
$m_{U}$ & 12.84 & de Vaucouleurs et al. (1991) \\
$m_{B}$ & 12.87 & de Vaucouleurs et al. (1991) \\
$m_{V}$ & 13.11 & Huchra (1977) \\
$M_{B}$ & -20.7 & above with $E(B-V)=0.04$ \\
IRAS 12 microns & $0.14 \pm 0.049 \mathrm{Jy}$ & Soifer et al. (1989) \\
IRAS 25 microns & $0.52 \pm 0.042 \mathrm{Jy}$ & Soifer et al. (1989) \\
IRAS 60 microns & $4.98 \pm 0.047 \mathrm{Jy}$ & Soifer et al. (1989) \\
IRAS 100 microns & $6.66 \pm 0.321 \mathrm{Jy}$ & Soifer et al. (1989) \\
$L_{\mathrm{FIR}}$ & $6.3 \times 10^{43} \mathrm{erg} \mathrm{s}^{-1}$ & IRAS 60 \& 100 micron flux after Hunter et al. (1989) \\
\hline
\end{tabular}

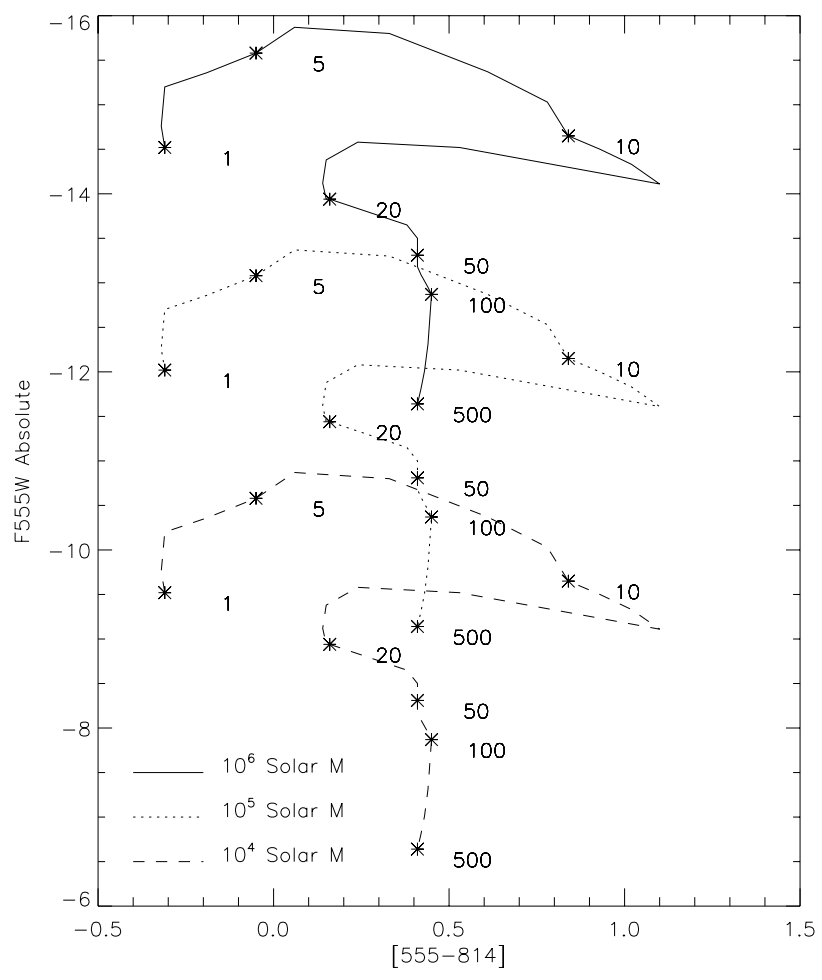

Fig. 1. This figure presents Starburst99 (Leitherer et al. 1999) model tracks illustrating the effect of changing the mass of the star cluster. All model tracks are for an instantaneous burst. Asterisks mark the time at 1, 5, 10, 20, 50, 100, and $500 \mathrm{Myr}$. Note that the only effect of a lower mass is to shift the tracks downward at constant color; stochastic effects are not included.

\section{Models}

We are using the Starburst99 evolutionary models (Leitherer et al. 1999) to compare our colors and magnitudes. We calculate a magnitude for each WFPC2 filter using standard response functions at the selected ages and metallicities. Clusters are represented by an instantaneous burst of $10^{6} M_{\odot}$ with an upper mass limit of $100 M_{\odot}$ and a lower mass limit of $1 M_{\odot}$. Varying

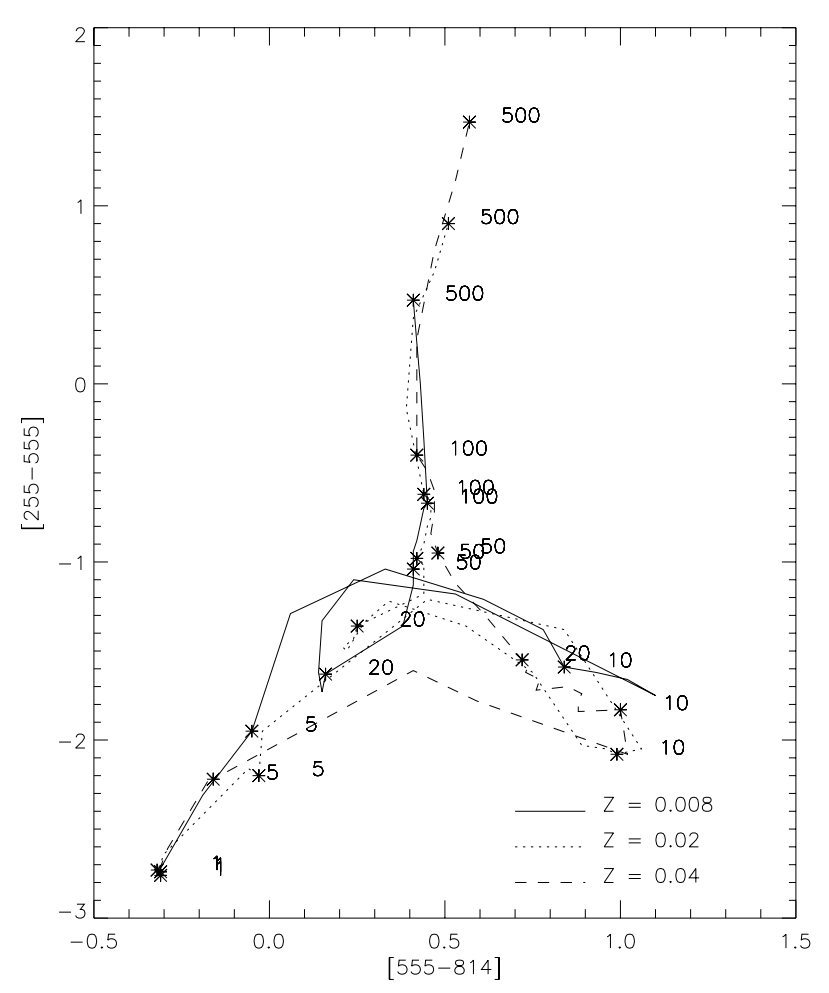

Fig. 2. This figure presents Starburst99 (Leitherer et al. 1999) model tracks illustrating the effect of changing metallicity. All model tracks are for an instantaneous burst of $10^{6} M_{\odot}$. Asterisks mark the time at 1 , 5, 10, 20, 50, 100, and $500 \mathrm{Myr}$.

the mass of the burst affects the magnitude as shown in Fig. 1, but not the colors. This means that our two-color comparisons are mass-independent, insofar as stochastic effects are not important in the real star clusters (see Lançon \& Mouhcine 2000). Varying the metallicity affects both the magnitudes and the colors; this effect is shown for a two-color diagram in Fig. 2. We do not account for stochastic effects, which should introduce a spread in colors at cluster masses below $10^{5} M_{\odot}$ (see Cerviño et al. 2002). 


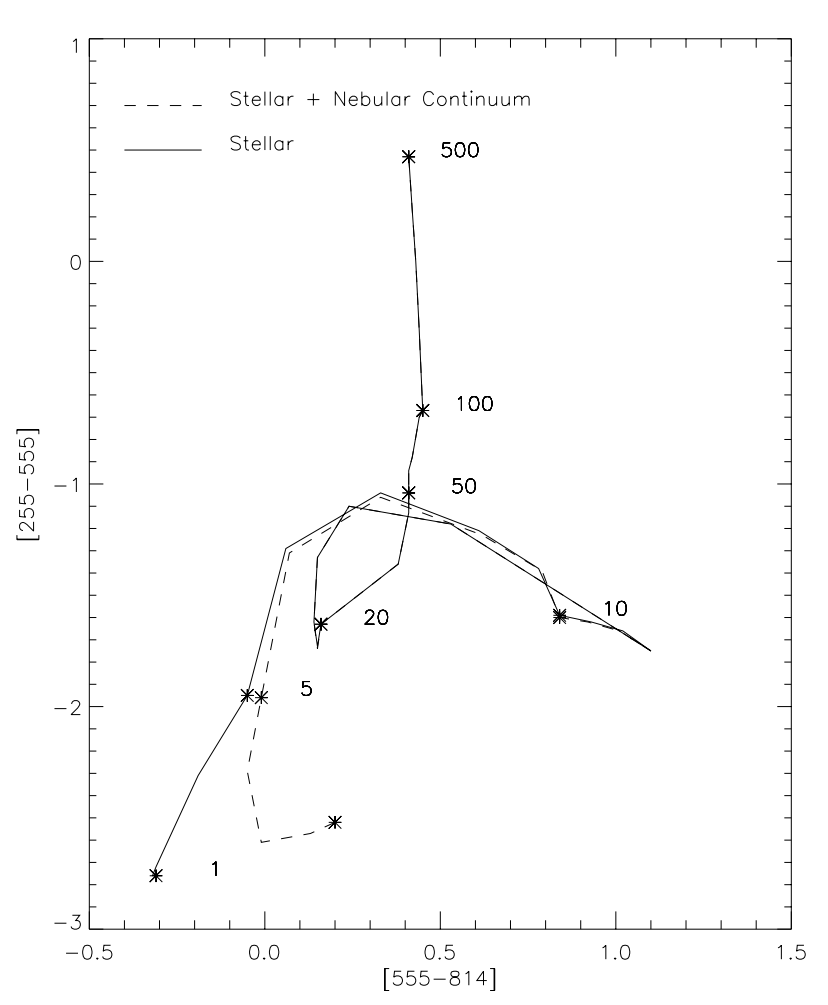

Fig. 3. This figure presents Starburst99 (Leitherer et al. 1999) model tracks illustrating the difference between models with only stellar emission and those with stellar and nebular continuum emission for an instantaneous burst of $10^{6} M_{\odot}$. Time steps are as for Fig. 2 .

For clarity, we have chosen to plot only a single metallicity track for comparison with the NGC 7673 star clusters, $Z=0.008$, or just under half $Z_{\odot}$. The abundance of this galaxy has been found to be $12+\log \mathrm{O} / \mathrm{H} \sim 8.6$ for the various clumps, slightly above that of the LMC (Duflot-Augarde \& Alloin 1982), which is confirmed by a reanalysis of the spectrophotometry reported by Gallagher et al. (1989).

\subsection{Nebular emission}

The models we have chosen to use include only stellar emission. Starburst99 also offers the opportunity to include nebular continuum emission, calculated by assuming that every photon below $912 \AA$ is converted into free-free and free-bound photons at longer wavelengths (nebular case B). Although the model assumptions may not apply, for comparison we show in Fig. 3 the variation in the two-color diagram for star cluster models including stellar emission only vs. stellar + nebular continuum. Here we can see that the difference is significant only for ages $<7$ Myr.

In young systems we also need to consider the influence of strong nebular line emission, as well as the weaker nebular continuum. Johnson et al. (1999) investigated the effects of nebular emission lines on WFPC2 star cluster colors. The main effect is due to strong emission lines of [OIII] and $\mathrm{H} \beta$ in the $\mathrm{F} 555 \mathrm{~W}$ filter. While the nebular continuum emission makes the models redder in [555-814], they found the line emission makes them bluer, and the difference can be large for ages $<10 \mathrm{Myr}$, depending on the strengths of the emission lines relative to a cluster's stellar continuum (e.g., Olofsson 1989; Zackrisson et al. 2001).

Unfortunately, the issue is not as simple as deciding whether to include nebular emission or not. For example, the nebular emission, both continuum and line, may be subtracted as part of the background if it extends smoothly beyond the cluster. So unless the nebular emission characteristics are known, e.g., from high angular resolution spectra, it is difficult to extract accurate ages from optical broad band colors of very young star clusters. Nevertheless, we proceed with fortitude, comparing our data with purely stellar continuum model tracks, aware that nebular effects may be important.

A rough estimate of the kind of effect that could occur can be estimated from the 10 arcsec circular aperture spectrum of Clump A $\mathrm{A}^{4}$ presented in Gallagher et al. (1989). The combined emission equivalent widths from the $\mathrm{H} \beta$ line (corrected for underlying absorption; as in Gallagher et al. 1989) and the [OIII] $\lambda 5007+\lambda 4959$ doublet is about $90 \AA$. This then would produce an excess brightness of $\approx 0.07 \mathrm{mag}$ in the $\mathrm{F} 555 \mathrm{~W}$ filter, and smaller offsets in the other two filters. Of course this emission correction would be an underestimate if the emission lines preferentially are associated with the individual star clusters. We can derive a different correction for this case by assuming all of the line emission is included in the photometry of the star clusters in Table 6 . In this case we find a substantial correction of 0.3 mag due to the emission lines in the F555W filter.

In summary, the figures subsequently found throughout this paper show only and always the model track for purely stellar emission from a $10^{6} M_{\odot}$ cluster at a metallicity of $Z=0.008$.

\section{Morphology}

Figure 4 is a mosaiced F555W image with a different scaling to illustrate lower surface brightness regions. Figure 5 presents ground-based $R$ and $\mathrm{H} \alpha$ images obtained with the WIYN 3.5-m telescope; the reader is referred to HG for information about these data. Here they are presented to show the clump designations by previous researchers, and for comparison with the WFPC2 images. Figure 6 shows the main body of NGC 7673 as observed by WFPC2 in the three filters: F255W, F555W, and F814W.

From previous ground-based images, five main clumps have been identified (Duflot-Augarde \& Alloin 1982). These are designated as Clumps "A-E"; here we refer to Clump A as the nuclear region, while Clumps B-F retain their original designations. Four of the clumps are strong $\mathrm{H} \alpha$ sources, and three of the clumps (B, C, and A/Nuclear) were observed in the Gallego et al. (1997) study of UCM galaxies. The H $\alpha$ luminosity ranges from $2-8 \times 10^{8} L_{\odot}$ in these regions. Both the nuclear region and Clump $B$ are strong non-thermal radio sources, with an inferred rate of 0.1 type II SN $\mathrm{yr}^{-1}$ over the last $30 \mathrm{Myr}$ (Condon \& Yin 1990).

${ }^{4}$ Clumps are labeled following the notation of Duflot-Augarde \& Alloin (1982). 


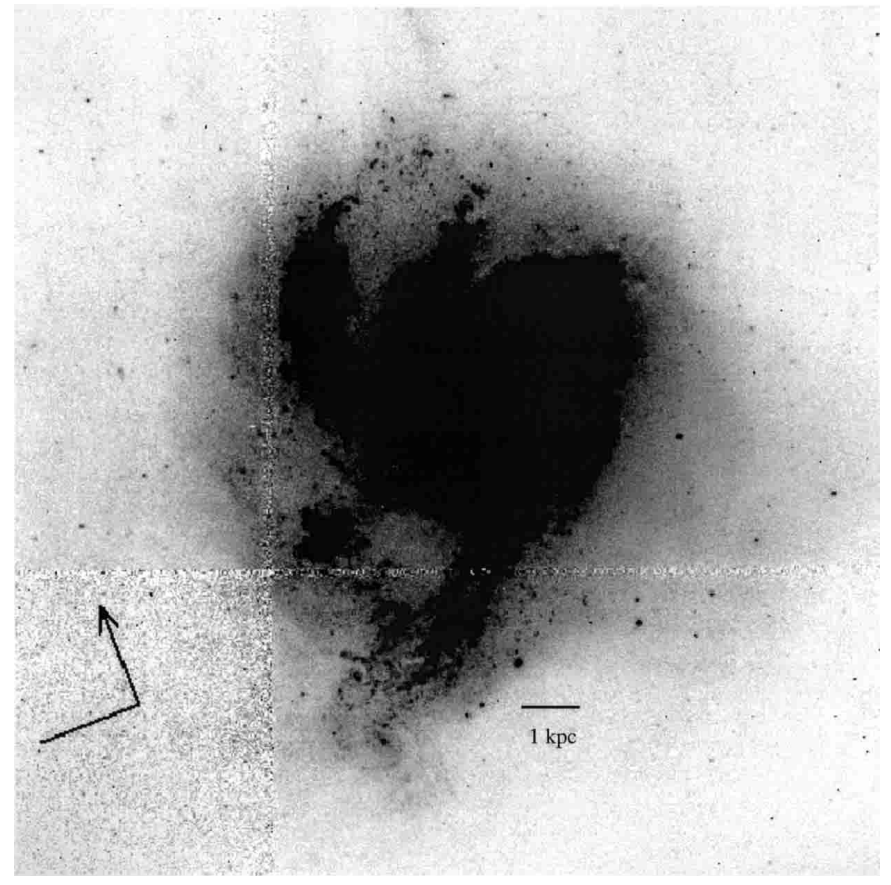

Fig. 4. F555W mosaiced image. The lines are boundaries of the WFPC2 CCDs; the galaxy is mainly in the WF3 CCD with extensions into WF2 and WF4. The arrow indicates North (arrowhead) and East.

The nuclear region (Clump A), has a pronounced bar shape, studded with luminous star clusters. The spiral pattern is most noticeable on the north-eastern side of the galaxy, with an arm of $\sim 2 \mathrm{kpc}$ in length emerging from the end of the bar to play host to the highest surface brightness UV region in this system, Clump B. Small, stubby features, which suggest weak spiral armlets, extend from the nuclear region on the opposite side of the bar, which are also dotted with star formation regions. Overall, the impression is of a disturbed, lopsided barred spiral, without a distinct nucleus.

\subsection{Color map and dust}

The color map shown in Fig. 7 was produced by dividing the F555W image by the F814W image, both in $\mathrm{DN} \mathrm{s}^{-1}$. This allows us to examine the distribution of dust and blue clusters in a qualitative way. From this we can see that the clumps are prominently "blue" in the color map, excepting Clump D. The dust is mainly concentrated in and around the nuclear region, which has a conspicuous dust lane cutting across the center, and an overall patchy appearance. There are weak spiral arm features delineated by the blue clusters and dark dust lanes.

Finally we note that in addition to the patchy dust extinction, faint but well-defined dust features extend in a "V" from a bright region in the nuclear clump (see Fig. 8). This peculiar structure may be the edges of a galactic chimney. The presence of a large scale outflow might be expected in a galaxy with a SFR as high as in NGC 7673.

\section{2. “Clump F”: No clump, just cluster}

The region of the galaxy labeled "Clump F" appears distinctly non-clumplike in our WFPC2 images. Instead of an extended star formation region composed of many clusters such as Clump B or Clump D, this region is dominated by a single object. The apparent F555W magnitude of this object is 19.5, and its inferred absolute magnitude is -14.0 . This is extraordinarily bright compared to young clusters in e.g. the Antennae (Whitmore et al. 1999). In fact there is only one cluster in the Antennae sample which approaches this luminosity, and even the dominant cluster in Hodge complex of NGC 6946 (which at -13.7 contributes $17 \%$ of the light, Larsen et al. 2002) is less luminous than this.

From Fig. 5, we can see that this object is embedded in a strong $\mathrm{H} \alpha$ emitting region. Due to the low resolution of the available $\mathrm{H} \alpha$ data, we cannot deduce the amount of nebular emission coming from this cluster. It is possible the structure of the emitting gas is complex, and the star cluster has blown out the gas in its immediate vicinity, allowing us to observe the unextincted stellar light. Closer inspection reveals that the structure observed in the F255W and F555W is not the same, but the source of this difference is unknown. Multiple stellar clusters, dust extinction, or inhomogeneous nebular emission could be responsible.

\section{The cluster population}

As stated in Sect. 2, we have two samples of star clusters: those detected in all three WFPC2 filters, and those only detected in the F555W and F814W filters. We will discuss each set separately, beginning with the three-filter set. For this set of objects we have greater age discrimination, and we will use this information to investigate cluster age spreads both within and between the clumps.

\subsection{F255W, F555W, and F814W}

We have detected 50 objects with our selection criteria in the three filters: 12 in the nuclear region, 8 in Clump B, 12 in Clump C, and 18 others not associated with a particular clump. The coordinates, colors, and F555W magnitudes are presented in Table 2, and Fig. 9 shows the objects overplotted on a F555W image as white dots with number labels. Two-color diagrams for these objects are shown separated by region in Fig. 10, with an overplotted Starburst99 cluster model track for $Z=0.008$, with ages in Myr. The reddening line is for $A_{v}=1.0$ using a Calzetti et al. (1994) extinction law for starburst galaxies. Mean [255-555] and [555-814] colors are presented in Table 3.

From the color map in Fig. 7, we would not expect all of the clusters to escape the effects of dust extinction. However, we might expect to see only weak dust effects in our sample, because we are heavily biased in favor of clusters which do not have significant reddening (for a discussion see Conselice et al. 2000b and Calzetti 2001). One magnitude of visual extinction makes a cluster approximately 2.4 mag fainter in our F255W filter. Thus we only expect to see the brightest, so the youngest and most massive, star clusters in this sample. 

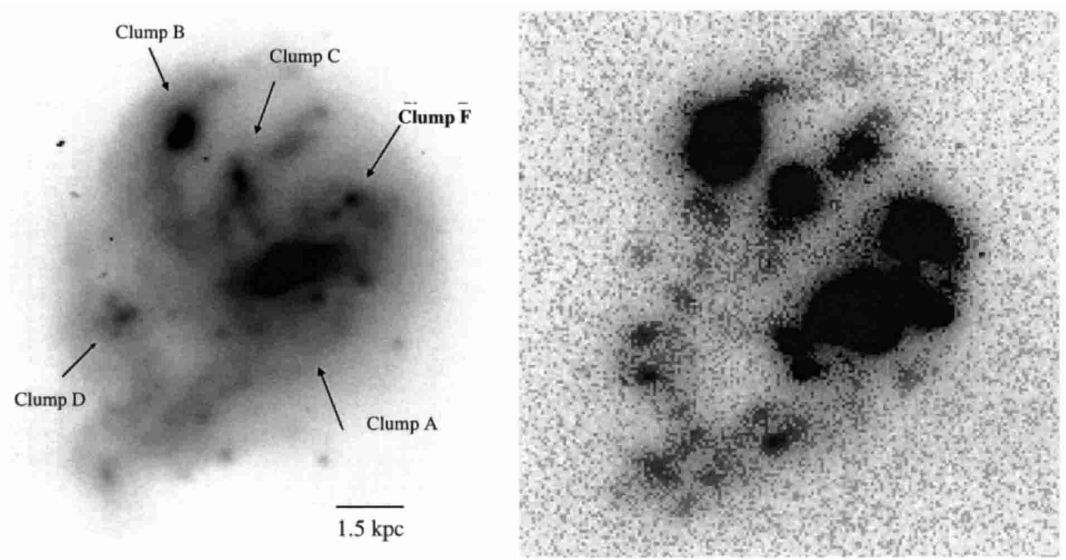

Fig. 5. Left: a ground-based WIYN telescope $R$-band image showing the main clumps, which are labeled for reference following the designations of Duflot-Augarde \& Alloin (1982). For both, North is up and East is to the left. Right: a WIYN H $\alpha$ image showing that most of the clumps are strong $\mathrm{H} \alpha$ sources. The exception is Clump D, which has instead regions with $\mathrm{H} \alpha$ emission to the NE and SE.
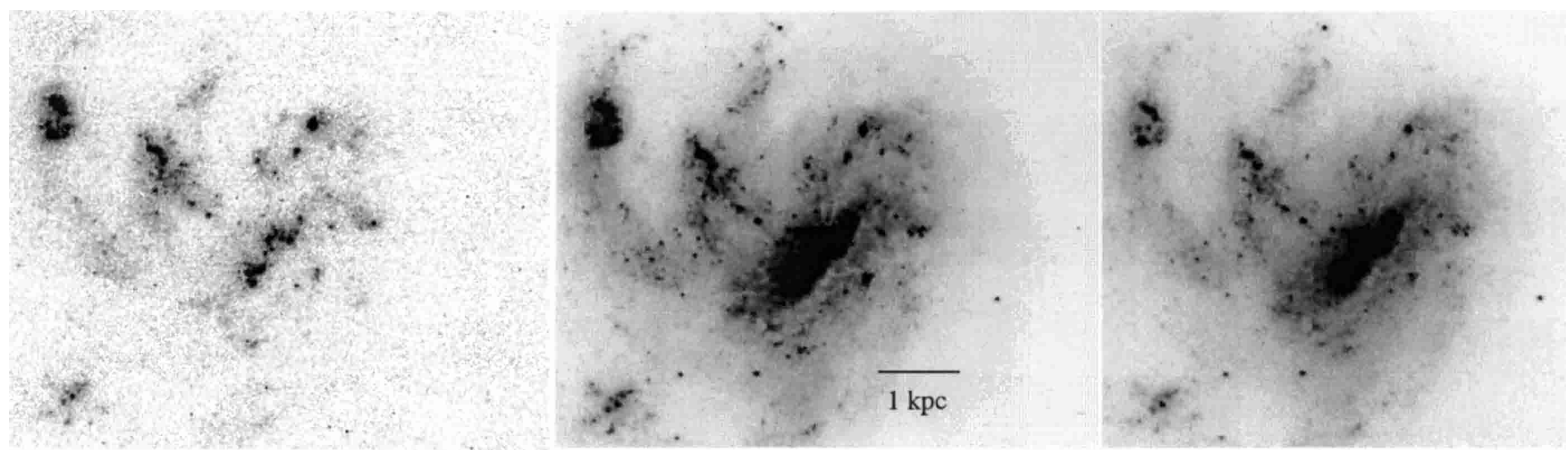

Fig. 6. HST WFPC2 images, from left to right: F255W, F555W, F814W. Orientation is as for Fig. 4.

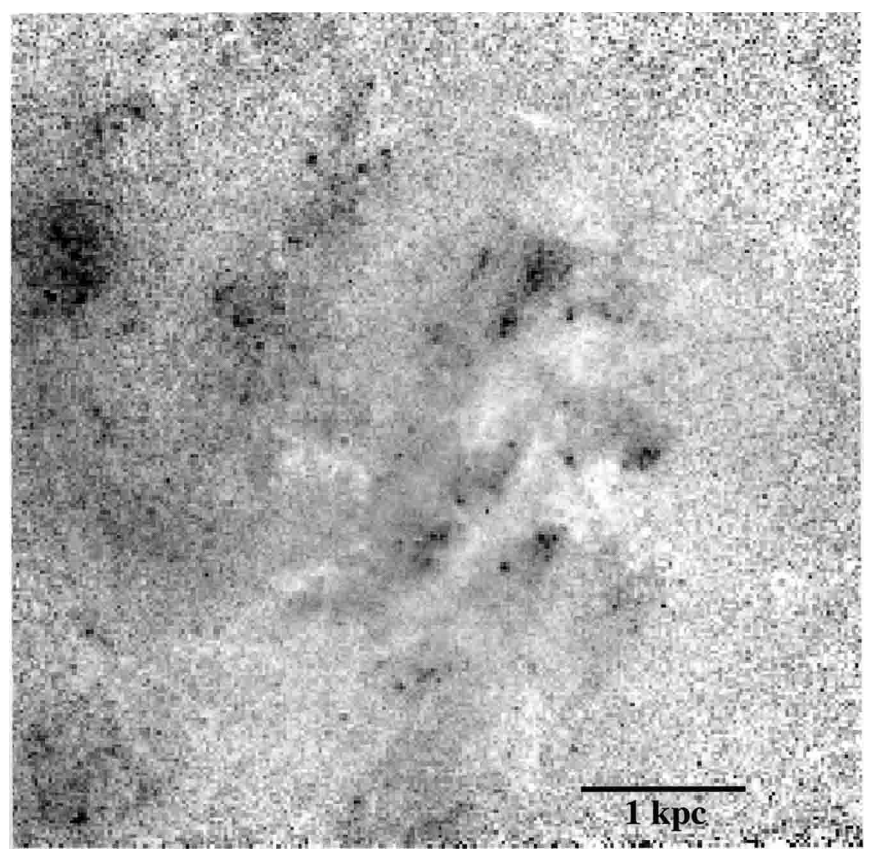

Fig. 7. A color map made from the F555W and F814W images shows bluer objects as darker, and redder as lighter. Orientation is as for Fig. 4. Clump B is prominent in the top left (NE) corner of this image; Clumps $\mathrm{C}$ and $\mathrm{F}$ are also very blue. This image also displays the complex small-scale dust structures associated with the nuclear region (Clump A).

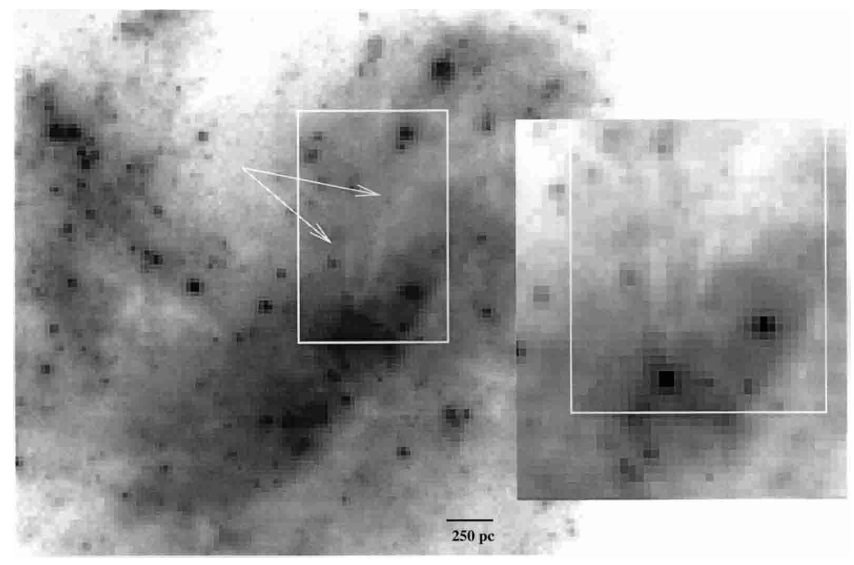

Fig. 8. This F555W filter image of the central part of NGC 7673 shows the pair of linear dust structures emanating from a bright source, which may be the nucleus. Orientation is as for Fig. 4.

At its most luminous, a $10^{6} M_{\odot}$ cluster is predicted to have a F255W magnitude of -17.9 at an age of $3 \mathrm{Myr}$, or an apparent $m(\mathrm{~F} 255 \mathrm{~W}) \approx 15.3$ at the distance of NGC 7673. With our selection criteria we are sensitive to $3 \mathrm{Myr}$ clusters down to $10^{4} M_{\odot}$, but only $10^{5} M_{\odot}$ clusters at $20 \mathrm{Myr}$, due to rapid fading with age. 

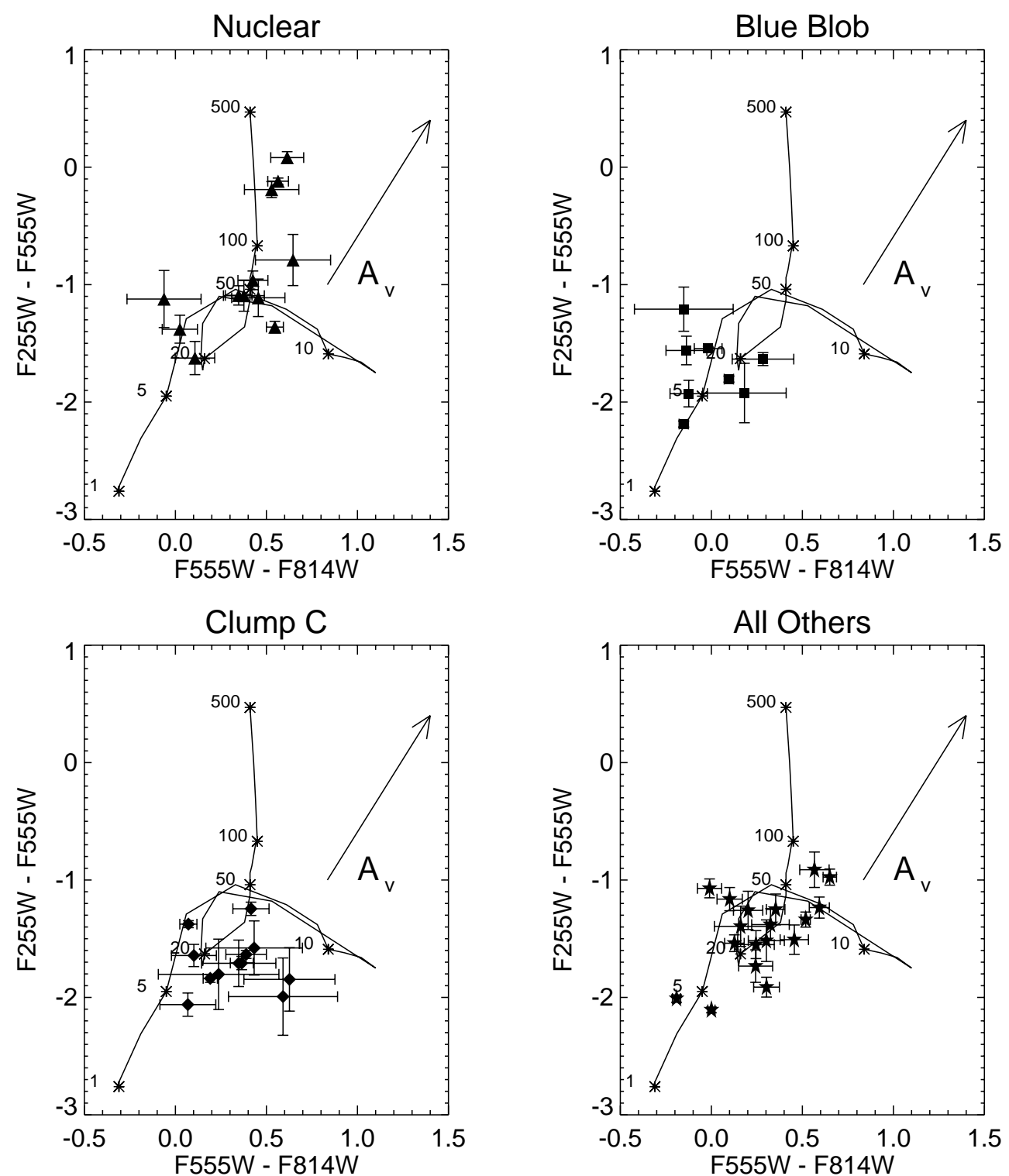

Fig. 10. Two color plots for the clusters. Filled stars mark ages of 1, 5, 10, 20, 50, 100, and 500 Myrs on a model track for purely stellar emmission (see Sect. 4.1). Clockwise from top left: nuclear, the "blue blob" which is Clump B, all other clusters not associated with a particular clump, and Clump C. The arrow indicates $A_{v}=1$. See discussion in the text.

\subsubsection{Nuclear region}

The nuclear region is unique as the only area significantly affected by dust in this galaxy. This complicates our interpretation of the colors, because of the degeneracy between age and reddening. The reddening line can place a cluster at $1 \mathrm{Myr}$ on the track anywhere from 2 to $100 \mathrm{Myr}$.

The nuclear clusters span a wide range of [255-555] and [555-814] colors. Three clusters (Numbers 4, 5, and 7) have blue [555-814] and [255-555] colors, and we suggest that these are young, $\sim 6$ Myr clusters suffering from little extinction. Four clusters (Numbers 1, 8, 11, and 12) concentrate near [255-555] -1.1 and [555-814] 0.45. This places them on the model track at $30-50 \mathrm{Myr} . A_{v}=0.5$ could make $20 \mathrm{Myr}$ clusters appear here, and $A_{v}=0.8$ could cloak 5 Myr clusters and move them to the $30-50 \mathrm{Myr}$ position on the two-color diagram. Three of the clusters (Numbers 2, 9, and 10) have [255-555] less than -0.2 and [555-814] 0.6. These could be $200 \mathrm{Myr}$ with a small amount of reddening, or 7-50 Myr with $A_{v}=1.0$.

Thus, we have 2 possible interpretations for the Clump A nuclear region star cluster population: (1) the clusters suffer little reddening $\left(A_{v}<0.2\right.$ everywhere $)$ in this region, and some of the clusters are young $\left(t_{\text {age }} \sim 6 \mathrm{Myr}\right)$, some are intermediate $\left(30 \mathrm{Myr}<t_{\mathrm{age}}<50 \mathrm{Myr}\right.$ ), and some are older $\left(t_{\text {age }}>100 \mathrm{Myr}\right)$. Or, (2), there is significant and variable extinction $\left(A_{v}=0.1-1.0\right)$ in this region, and all of the clusters are $\sim 7$ Myr. Additional information, such as high angular resolution spectra (e.g., Glazebrook et al. 1999), is needed to resolve this issue. 
Table 3. Mean [255-555] and [555-814] cluster colors for the three-filter selected sample.

\begin{tabular}{lccccc}
\hline \hline Region & Number of Members & Mean [255-555] (mag) & Stddev & Mean [555-814] (mag) & Stddev \\
\hline Nuclear & 10 & -0.90 & 0.54 & 0.38 & 0.24 \\
Clump B & 8 & -1.72 & 0.30 & 0.00 & 0.17 \\
Clump C & 12 & -1.70 & 0.23 & 0.32 & 0.19 \\
No Region & 18 & -1.43 & 0.33 & 0.23 & 0.30 \\
\hline
\end{tabular}

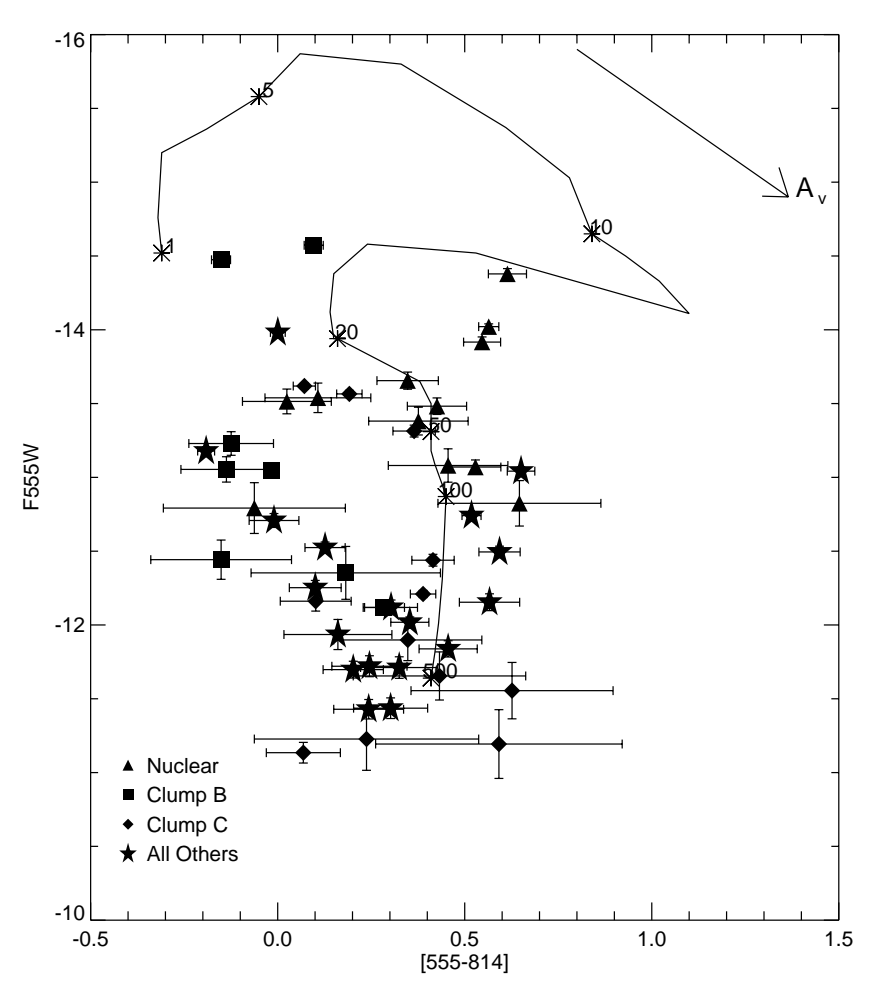

Fig. 11. Magnitude-color plot for the 3-filter selected clusters shown in Figs. 9 and 10. The 12 nuclear clusters are represented by triangles, the 8 Clump B clusters by squares, the 12 Clump C clusters with diamonds, and the others not associated with a particular region with stars. The model track is for an instantaneous burst of $10^{6} M_{\odot}$ with $Z=0.008$. With an assumption of age, we can shift the model track vertically and derive a mass for the cluster based on its F555W luminosity. The age assumption is most secure for the Clump B clusters ( $<7 \mathrm{Myr}$ ); thus the brightest clusters in Clump B have masses around $5 \times 10^{5} M_{\odot}$.

\subsubsection{Clump B: The blue blob}

From the color map we find that this region has little, if any, reddening, which should simplify our interpretation of the cluster colors. Looking at Fig. 10, we can see immediately that almost all the objects are consistent with ages $<6 \mathrm{Myr}$. The colors for 2 of the clusters (Numbers 3, and 5) are 0.1-0.2 mag too blue in [555-814], which could indicate contamination from nebular emission lines. We can easily mimic this effect by adding emission line fluxes to the F555W model magnitude. The clusters then overlap the models at ages 3-6 Myr. Three of the clusters (Numbers 1, 4, and 6) are consistent with ages $<4$ Myr with a few tenths of a magnitude of visual extinction.

If we assume an age for the clusters, we can use the inferred absolute F555W magnitude to estimate the masses.
The clusters range from -12 to -14.5 , and assuming an age of $5 \mathrm{Myr}$, they span a range in mass of $5 \times 10^{4}$ to $5 \times 10^{5} \mathrm{M}_{\odot}$.

\subsubsection{Clump C}

This region is complicated by a diffuse background in the F255W filter, indicating the presence of many faint F255W sources below our detection limits. This is likely to be the case in all regions, however here there is a lack of very massive clusters as seen in the nuclear region and Clump B, which would prevent the detection of such a background.

The Clump $\mathrm{C}$ region does not match the models; the [555-814] colors are too red for their [255-555] colors (see Fig. 10). However, Clump $\mathrm{C}$ is a strong $\mathrm{H} \alpha$ source, so we expect it to be young. A possible explanation for the colors is that we are over-subtracting a strong nebular background. This would make the $\mathrm{F} 555 \mathrm{~W}$ magnitude too faint. If we assume this is the case, the [555-814] color gets bluer and the [255-555] gets redder, moving them onto the model track around $6 \mathrm{Myr}$. The spread in F555W magnitudes is comparable to that of Clump B, but almost a magnitude fainter. For an age of $6 \mathrm{Myr}$, we estimate the star cluster masses lie between $2 \times 10^{4}$ and $2 \times 10^{5} M_{\odot}$.

\subsubsection{Other F255W sources: "Clump F" and the central cluster of Clump D}

What was designated as Clump F by previous researchers, is clearly dominated by a single star cluster, number 16 of the "no-region" clusters in Table 2. This object matches the model for a cluster with an age of 4-5 Myr, with perhaps a small over-subtraction of nebular emission. The sibling to this cluster, number 14 of the "no-region" objects, is also consistent with an age of 4-5 Myr if we consider a contribution of $0.1 \mathrm{mag}$ in F555W from nebular line emission. With this small nebular correction and the age assumption of $5 \mathrm{Myr}$, the clusters have masses of $3 \times 10^{5} M_{\odot}$ and $1 \times 10^{5} M_{\odot}$.

The central cluster of Clump D (Number 1) falls slightly below the model track for a cluster with an age of 9-13 Myr, and its companion (Number 2) is off a bit farther, although along the reddening vector. Assuming the photometry is reasonably accurate and the models are not in error, these clusters can move to this area of the two-color plot only by being very young, with ages of $1 \mathrm{Myr}$, and having more than $1 \mathrm{mag}$ of visual extinction. However, they are unlikely to be younger than $10 \mathrm{Myr}$, considering the lack of $\mathrm{H} \alpha$ emission (see Sect. 4). Also, we expect little to no reddening from the color map. If we consider solar metallicity instead of $Z=0.008$, the model track moves blue-ward in [255-555], eliminating any disagreement 
with the data. But it seems unlikely that the metallicity in this region should be greater than that of the nuclear region. Rather, it seems more probable that Clump D has a lower metallicity.

We also consider the possibility that the model tracks are in error. Allowing for the well-known uncertainty in redsupergiant stellar evolutionary tracks (Langer \& Maeder 1995; Mayya 1997; Origlia et al. 1998; Leitherer et al. 1999), especially at metallicities less than solar, it seems possible that the [255-555] color may be $\sim 0.2 \mathrm{mag}$ too red. With all these possible factors, we prefer the explanation of a 9-13 Myr cluster with little to no extinction.

An age of 9-13 Myr also explains something noteworthy in the $\mathrm{H} \alpha$ image. $\mathrm{H} \alpha$ emission is not seen in the center of Clump D, but at the edges (NE and SE of the central cluster). Could this be evidence for propagating star formation? Clusters are detected in these regions in the F555W and F814W filters (presented in Sect. 6.2.4), and while their colors are not remarkably blue, they are consistent with ages of 7-8 Myr. This is interesting, although certainly not conclusive.

\subsection{F555W and F814W}

Now we turn to the set of clusters selected in the F555W and F814W filters, where we have detected 268 candidate star clusters with our selection criteria. Splitting this population into regions as we did previously, we find 20 Clump A star clusters, 14 clusters in Clump B, and 18 in Clump C. Here we also consider Clump D, which was too faint at 255, but in two filters has 11 members. Thus, there are 200 candidate star clusters, mostly relatively faint, which are not associated with a particular clump.

The color magnitude plots for the clusters separated by region are shown in Fig. 13, and the color magnitude diagram for the entire cluster sample is shown in Fig. 14. The clusters span a wide range of colors, with no apparent trend with luminosity. At the bright end are clusters in Clump B and the nuclear region. The cluster population not associated with a particular region starts to appear about two magnitudes fainter than these. This means that the most massive clusters are born in the clumps, although cluster formation is occurring throughout the galaxy.

For comparison with studies of other star-forming galaxies, we convert our [555-814] colors to standard Johnson $V$ and $I$ using the transformations found in H95b. The histogram of cluster colors is shown in Fig. 12, using a bin size of 0.15 mag. Mean $V-I$ colors are shown in Table 4, along with the mean [555-814] colors.

The mean color of this distribution, 0.42 , is blue, and comparable to the young cluster population in the Antennae (0.4, age $<30 \mathrm{Myr}$, Whitmore et al. 1999), the blue cluster population of NGC 7252 (0.4, age 30 Myr, Miller et al. 1997), and the cluster population of the 4 Myr starburst in NGC 1741 (0.4, Johnson et al. 1999). This suggests a similarly young age for the cluster population presented here. It is clearly younger than the cluster population of NGC $3921(0.59$, age 250 Myr, Schweizer et al. 1996).

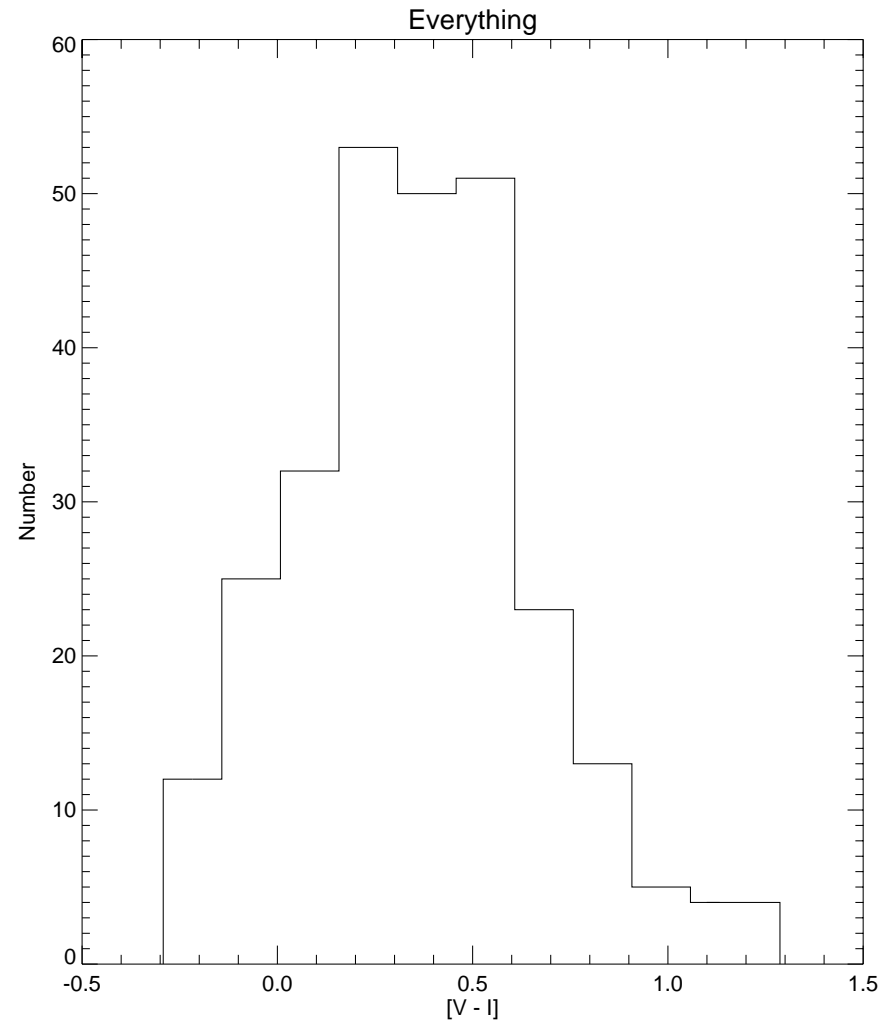

Fig. 12. Histogram for the 268 clusters in the final F555W and F814W sample, transformed to Johnson $V$ and $I$. The mean color of this distribution is 0.42 , with a dispersion of 0.29 .

\subsubsection{Nuclear}

The mean color of this region is redder than the cluster population as a whole. Looking only at the color map, we would suspect this to be the case due to dust extinction. From Fig. 13, we can see this is indeed what is happening. There is a pronounced color-luminosity trend, where the faintest clusters are the reddest $([555-814] \sim 0.65)$. There are two possible causes for a color-luminosity trend, age and reddening. We can rule out an aging effect since the [555-814] color of a cluster is nearly constant as it ages. Therefore, the observed trend must be due to dust extinction. There are three important exceptions to this trend; the three brightest clusters have red colors ([555-814] > $0.5)$. Either these are old and very massive clusters, or they are very young and have yet to destroy their dusty natal cocoons. This can only be determined from spectroscopy, or, perhaps from high angular resolution images in emission lines that would reveal connections to the surrounding ionized gas.

\subsubsection{Clump B: The blue blob}

The color spread in this region is small, approximately $0.2 \mathrm{mag}$ in [555-814]. However, there is a tendency for less luminous clusters to be redder, suggesting the effects of a small amount of reddening, requiring a correction for $\sim A_{v}=0.3 \mathrm{mag}$. If we make this small correction to the faint clusters, the color of this region becomes nearly zero in [555-814], and the color spread becomes even smaller, $\sim 0.15 \mathrm{mag}$. Now we can say something about the formation timescale of this region. From a 

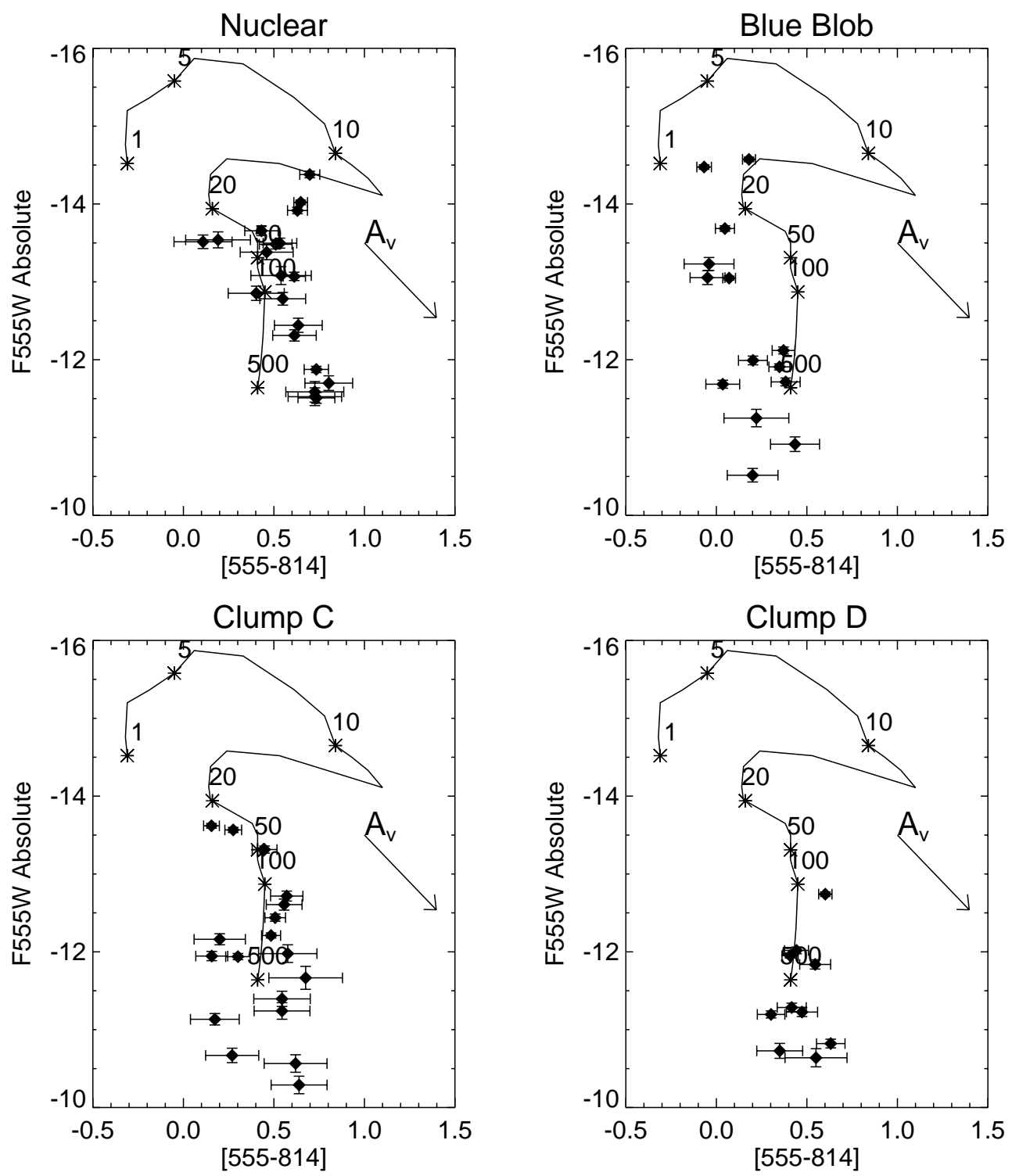

Fig. 13. Magnitude-color plots for the clusters separated by region. The overplotted model track is for an instantaneous burst of $10^{6} M_{\odot}$, with ages of 1, 5, 10, 20, 50, 100, and 500 Myr marked with asterisks. A purely stellar emission model is used, i.e. nebular contributions are omitted (see Sect. 4.1). The track shifts down by $2.5 \mathrm{mag}$ for $10^{5} M_{\odot}$, with no change in the F555W-F814W color (see Fig. 1).

dynamical point of view, we would not expect a region of this size, radius $\sim 0.4 \mathrm{kpc}$, to have an age spread less than $3 \mathrm{Myr}$ (using $v=150 \mathrm{~km} \mathrm{~s}^{-1}$ ). But for this small range in color, the age spread cannot be more than $3 \mathrm{Myr}$, could be smaller, and is only compatible with the models if the entire region is younger than 6 Myr. Thus, we conclude that the clusters in Clump B are less than $6 \mathrm{Myr}$ with age spread $\leq 3 \mathrm{Myr}$. With this age, cluster masses range from $5 \times 10^{5}$ to $5 \times 10^{3} M_{\odot}$.

\subsubsection{Clump C}

The mean cluster color in this region is redder than that of Clump B, closer to that of Clump D, but with a larger color spread. Only for the brightest clusters does there appear to be a trend of color with luminosity. The mean color of this region suggests an age less than $50 \mathrm{Myr}$, although the colors of the three-filter sample suggests that at least some of the clusters are less than $6 \mathrm{Myr}$. As we saw for the three-filter sample, there may be complicating nebular effects in this region.

\subsubsection{Clump D}

The clusters associated with Clump D all have roughly the same color, but a range of luminosities. This could mean that they have the same age and different masses, or a small range in mass, and ages $<30 \mathrm{Myr}$ with a spread. With a radius of approximately $200 \mathrm{pc}$, an age spread greater than $1.3 \mathrm{Myr}$ is expected. This region is significantly fainter than the other star forming regions in this galaxy, with the brightest member at only 20.7 mag at F555W, or -12.7 in absolute magnitude. For the derived age of 9-13 Myr (as discussed in the previous subsection), we find a mass of $2 \times 10^{5} M_{\odot}$. 
Table 4. Mean $V-I$ and 555-814 cluster colors for the two-filter selected sample.

\begin{tabular}{lcccc}
\hline \hline Region & Number of Members & Mean $[V-I](\mathrm{mag})$ & Mean [555-814] (mag) & Stddev (mag) \\
\hline Nuclear & 20 & 0.47 & 0.56 & 0.18 \\
Clump B & 14 & 0.07 & 0.17 & 0.17 \\
Clump C & 18 & 0.33 & 0.43 & 0.18 \\
Clump D & 11 & 0.40 & 0.49 & 0.13 \\
\hline All Clusters & 268 & 0.42 & 0.52 & 0.29 \\
\hline
\end{tabular}

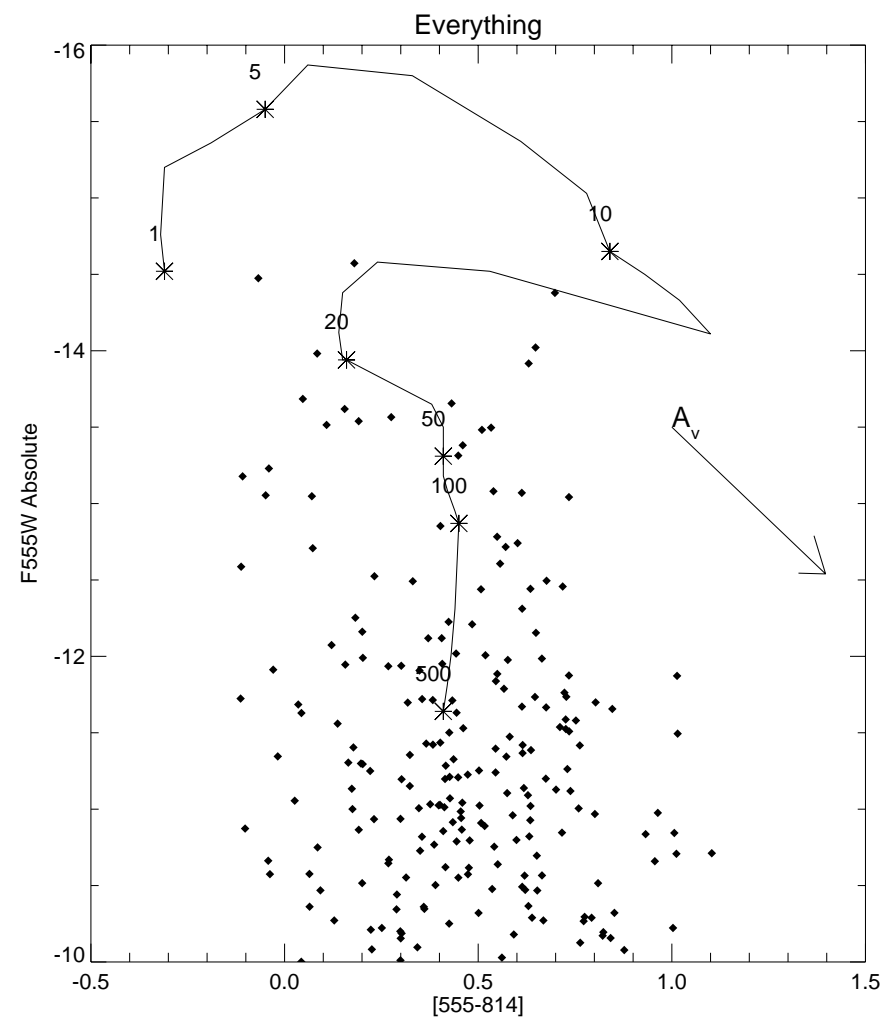

Fig. 14. F555W magnitude and [555-814] magnitude color plot for the 268 two-filter selected clusters. The overplotted model track is for an instantaneous burst of $10^{6} M_{\odot}$, with ages of $1,5,10,20,50,100$, and $500 \mathrm{Myr}$ marked with asterisks. The track shifts down by $2.5 \mathrm{mag}$ for $10^{5} M_{\odot}$, with no change in the [555-814] color (see Fig. 1).

\section{Clump properties}

To investigate the clumps in more detail, we have performed photometry with POLYPHOT for the nuclear region, and APPHOT with radii of 20, 22, and 19 pixels for Clumps B-D. The results are shown in Table 5. For the [255-555] numbers, we have used the three-filter selected objects, while for the [555-814] set, we have used the two-filter selected set of objects.

We can immediately see that the nuclear region is the reddest in both colors. Regardless of the age of the latest burst in this region, we expect this to be the case from the color map. Clump B is bluest in [555-814] color, and, perhaps surprisingly, Clump $\mathrm{C}$ is bluest in [255-555] color.

We can use these measurements to estimate the efficiency of cluster formation by calculating the flux from clusters vs. the total flux from the region; these results are shown in Table 6.
Measurements of the fraction of light from clusters at $2200 \AA$ in a sample of starburst galaxies found an average of $\sim 20 \%$ (Meurer et al. 1995). The numbers here are similar, and range from $13 \%$ near $I$ for the nuclear region, up to $33 \%$ for the midUV flux coming from clusters in Clump B. At F555W, the total flux in clusters compared to the total galaxy flux is $7 \%$.

The F255W filter gives us information about the stellar emission only, and primarily about the young, massive stars which dominate at this wavelength. One consequence of this is that a measurement of the fraction of the F255W flux coming from the clusters compared to the overall flux gives a direct measurement of the massive star formation occurring below our cluster detection limits. In contrast, the F555W and F814W filters tell us about the combination of stellar and nebular emission.

Clump B has $33 \%$ of its UV emission coming from only 8 clusters, but this number drops to $22 \%$ of visual light coming from 14 clusters, and $20 \%$ at F814W. Clump C has $22 \%$ of its UV emission from 12 clusters, but this number rises to $26 \%$ at F555W and F814W with a total of 18 clusters. We interpret this as evidence for an underlying stellar population in Clump B, which is absent in Clump C. Although Clump B is a strong $\mathrm{H} \alpha$ source, we can rule out nebular emission not associated with the clusters, as this would make the [255-555] and [555-814] colors too blue and too red, respectively.

\section{Discussion}

\subsection{Clump summary}

We have shown that the clumps are composed of many bright super star clusters, and that many of these are less than $6 \mathrm{Myr}$. This conclusion remains, despite the difficulties in deriving ages below $7 \mathrm{Myr}$ due to nebular emission and unknown geometry. The nuclear region contains a bar of star clusters, some of which are certainly young, and others which may be old, but are likely also young and suffering from dust extinction.

Clump B is a luminous, high surface brightness, and very young region, which has some evidence for an older, underlying stellar population. The young star clusters have ages less than $6 \mathrm{Myr}$, with masses ranging from $5 \times 10^{4} M_{\odot}$ to $5 \times 10^{5} M_{\odot}$. For these 8 clusters, we have a combined mass of approximately $8 \times 10^{5} M_{\odot}$.

We have shown in Sect. 7 that $33 \%$ of the flux at F255W is coming from these clusters, thus $2 / 3$ of the massive star formation is below our detection limits. We can use this to estimate the SFR in this region over the last $6 \mathrm{Myr}$, if we assume a constant $M / L$. If in the $1 / 3$ of the light that we observe we have 
Table 5. Photometric properties of the clumps.

\begin{tabular}{lccccc}
\hline \hline Region & F255W & F555W & F814W & 255-555 & 555-814 \\
\hline Nuclear & 14.22 & 14.84 & 14.23 & -0.62 & 0.61 \\
Clump B & 14.56 & 15.81 & 15.64 & -1.25 & 0.17 \\
Clump C & 14.91 & 16.48 & 16.07 & -1.57 & 0.41 \\
Clump D & 16.33 & 17.31 & 16.89 & -0.98 & 0.42 \\
\hline
\end{tabular}

Table 6. Fraction of flux from clusters vs. total flux.

\begin{tabular}{lccc}
\hline \hline Region & F255W & F555W & F814W \\
\hline Nuclear & 0.16 & 0.14 & 0.13 \\
Clump B & 0.33 & 0.22 & 0.20 \\
Clump C & 0.22 & 0.26 & 0.26 \\
Clump D & $\ldots$. & 0.16 & 0.17 \\
\hline
\end{tabular}

$8 \times 10^{5} M_{\odot}$, and we are missing $\sim 2 / 3$ of the massive stellar flux, this implies that the total mass formed in this region is $\sim 2.4 \times 10^{6} M_{\odot}$. With a radius of $0.4 \mathrm{kpc}$, Clump B then has a SF intensity of $0.8 M_{\odot} \mathrm{yr}^{-1} \mathrm{kpc}^{2}$. This incredible SF density puts it in the league with the most vigorously star-forming galaxies in the universe. Lanzetta et al. (2002) found objects at $z \geq 3$ with similar SF intensities over significantly larger areas than that of Clump B. However, Clump B still stands out as a large and intense extra-nuclear star forming site that can provide insights into the consequences of intense star formation on relatively large spatial scales.

Clump $\mathrm{C}$ is undoubtably young judging from its blue overall [255-555] color and those of the composing clusters. It may have embedded clusters judging from the combination of $\mathrm{H} \alpha$ emission and the anomalous cluster colors in our two-color diagrams.

Clump D is composed of fainter clusters, pointing to an older age and possibly less massive clusters. The derived age of the brightest cluster is between 9 and $13 \mathrm{Myr}$, and there are regions to the $\mathrm{NE}$ and $\mathrm{SE}$ with $\mathrm{H} \alpha$ emission, characteristic of young star formation regions, i.e. those with ages of less than 10 Myr. We suggest that this is evidence of propagating star formation, which should be followed up with high spatial resolution spectroscopy.

Clump $\mathrm{F}$ is not a clump but an $\mathrm{H}_{\text {II }}$ region dominated by a single cluster with an age of 4-5 Myr. This object is extremely bright, and the disparity between the F255W and F555W structures indicates smaller scale structure than we can resolve associated with dust or multiple ages.

The clumps in NGC 7673 appear to represent a step beyond normal $\mathrm{OB}$ associations in terms of their hierarchy of compact, gravitationally bound objects. In a simple OB association, the rare massive stars, some of which are either binary or multiple systems, define the upper end of the hierarchy. They contain only a modest fraction of the stellar mass, and are surrounded by more numerous, less massive single and multiple stars, which contain most of the mass.

In starburst clumps, compact star clusters define the top of the compact, bound mass distribution, and contain a significant fraction (more than 16\%) of the recently formed stars (see Sect. 7). These in turn are embedded in more diffuse fields of massive stars, which resemble rich examples of OB associations. Since high redshift galaxies also show signatures of starburst clumps, this mode of star formation, while rare in the current epoch, may have played an important role in building galaxies. As one of the nearest optically accessible clumpy starburst galaxies, NGC 7673 provides an excellent opportunity for further exploration of intense, large scale star formation.

\subsection{Starburst picture: Age of the current burst}

HG (1999) discussed the starburst trigger in detail, concluding that either the burst was triggered by an interaction with its companion, NGC 7677, or by the consumption of a small galaxy less than $10 \%$ of its mass in a minor merger. Neither of these scenarios can be ruled out, but in both cases the model must be able to account for the following:

1. The H I map of this pair shows a few appendages to the disk of NGC 7673, and a small extension from NGC 7677 pointing toward its companion, but otherwise is surprisingly regular.

2. NGC 7673 has an outer optical shell. This type of feature is usually associated with merger candidate E, S0, and only a few Sa galaxies (Schweizer \& Seitzer 1988), but has also been found around the starburst galaxy NGC 3310, which is thought to be triggered by a minor merger (Mulder \& van Driel 1996). Theoretical work indicates, however, that interaction scenarios other than mergers can produce arc-like features in outer disks like the one seen in NGC 7673 (Hernquist \& Quinn 1989; Hernquist \& Spergel 1992; Howard et al. 1993).

3 . There is a difference in timescale between the formation of this outer disk ripple and the very young starburst occurring in the inner disk. The outer disk "ripple" is characteristic of the late phases of an interaction, yet the starburst remains quite active. For example, if the starburst is associated with a close passage of NGC 7673's disturbed neighbor, NGC 7677 at a projected distance of $95 \mathrm{kpc}$, then the triggering event probably took place several hundred Myr in the past.

That $\mathrm{O}$ stars are present through out the galaxy is clear from the prominence of $\mathrm{H}$ II regions, indicating star formation within 


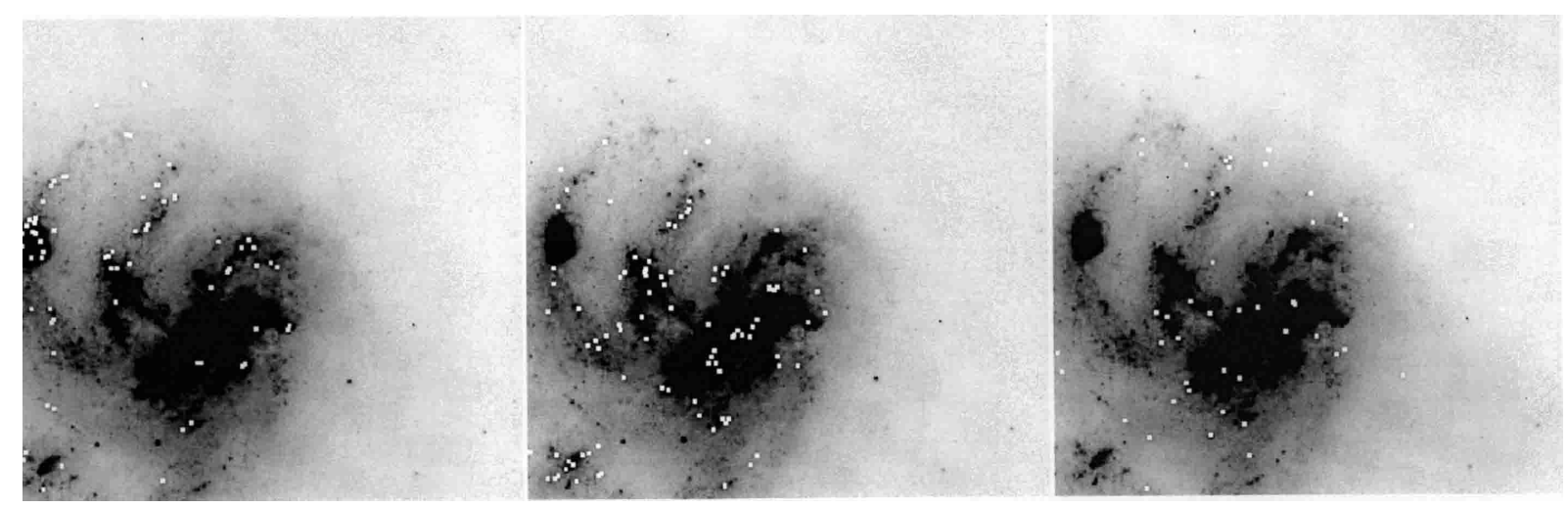

Fig. 15. The two-filter cluster sample within the main galaxy body. Orientation is as for Fig. 4. From left of right: blue clusters ([555-814] $<$ $0.4)$, neutral $(0.4<[555-814]<0.7)$, and red $([555-814]>0.7)$. The field of view for each panel is $40 \mathrm{arcsec}$, or $10 \mathrm{kpc}$ at the distance of NGC 7673.

the last 10 Myr (see HG). Duflot-Augarde \& Alloin (1982) also found evidence for an underlying older stellar population within the nuclear region from a weak $400 \mathrm{~nm}$ continuum break and Balmer absorption underlying the strong emission lines. However, the digital spectrum of the nuclear region taken by Gallagher et al. (1989) suggests that the $4000 \AA$ "break" is probably due to a reddened younger population. DuflotAugarde \& Alloin also found weak evidence from the $G$ band for the presence of an evolved stellar component in Clump B. This is supported by the fraction of flux at F555W from cluster as opposed to F255W. So the starburst history in this galaxy is complex.

Our two-color diagrams are useful in age-dating the brightest regions, although the rapid fading and sensitivity to dust of the F255W flux means we have only a small number of the entire cluster sample for this type of investigation. With this we find evidence for very young, $<6$ Myr star clusters in Clump B, Clump $\mathrm{C}$, and the nuclear region.

The color magnitude diagram for the entire cluster population shows a broad range in [555-814] and $V-I$ color, spanning the allowed model color range fully. It is difficult to constrain ages with only this color, although we do note that the mean is comparable to other starburst systems with burst ages less than $30 \mathrm{Myr}$ (such as the Antennae and NGC 7252), and one as young as 4 Myr (NGC 1741).

An overview of the distribution of cluster ages is illustrated in Fig. 15. This shows the bluest star clusters in [555-814], whose intrinsic colors imply ages of $<20 \mathrm{Myr}$, are concentrated in the blue blob, Clump B, with some presence in Clumps $\mathrm{C}$ and $\mathrm{F}$. These objects avoid the inner parts of the starburst, yet Clump A also displays strong $\mathrm{H} \alpha$ emission (see Fig. 5). The possible conclusions are that the $\mathrm{H}$-ionizing stars in Clump A are distributed in the field rather than in compact star clusters, or dust is an important factor.

The middle panel of Fig. 15 shows star clusters with colors consistent with ages of about 20-30 Myr up to 1 Gyr if they are unreddened or moderately reddened younger star clusters. Note these are absent in Clump B, where we see little evidence for dust; Clump B apparently is a young feature. The combination of "blue" and "neutral" star clusters delineate the main features of NGC 7673. The strong luminosity bias that favors observation of younger star clusters, the coincidence between the locations of these clusters and $\mathrm{H} \alpha$ emission, and the results from the F255W filter observations lend additional support to the view that the clumps in NGC 7673 are relatively young, with ages of $\leq 50$ Myr.

In this interpretation the upper age bound is set by Clump $\mathrm{D}$, which has weak $\mathrm{H} \alpha$ emission, faint star clusters with similar colors, and comparatively red global colors (Table 5). The third panel of Fig. 15 shows cluster candidates which either are highly reddened and young, luminous objects, or have colors of star clusters with ages of 1 Gyr or more. We are encouraged that this poorly defined sample of redder objects is not associated with the main clumps. Whatever the red objects are (possibly a mixture of background galaxies, clusters, and objects with large color errors), they do not seem to be associated with the ongoing starburst.

Using the clump colors in Table 4, we can estimate ages, which will lie between the values predicted by instantaneous and continuous SFR models. We again use the Starburst99 models. Clump B then must predate the red supergiant flash, and is younger than about 8-10 Myr. Clump C is 10-15 Myr old, while Clump D is $15-50 \mathrm{Myr}$ in age. These ages refer to the mean stellar population age. They are not corrected for any internal reddening, and including these would reduce the estimated ages.

\subsection{Evolution of the starburst clumps}

Huge regions of active star formation, such as those seen in NGC 7673, presumably reflect the presence of supergiant complexes of gravitationally bound interstellar gas clouds. These can form when a galactic disk becomes Jeans unstable. The Jeans mass for a rotating gaseous disk scales as $M_{\mathrm{J}} \propto{\overline{\sigma_{\mathrm{g}}}}^{2} / \mu_{\mathrm{g}} \propto$ $\mu_{\mathrm{g}}^{3} Q_{\mathrm{g}} / \kappa^{3}$, where $\mu_{\mathrm{g}}$ is the gas disk's surface density, $\overline{\sigma_{\mathrm{g}}}$ the velocity dispersion, $\kappa$ the orbital epicyclic frequency, and $Q_{\mathrm{g}} \sim 1$ the Toomre disk stability parameter (Elmegreen et al. 1993; Noguchi 1999). Galaxy interactions favor the formation of super cloud complexes in two ways: (1) They can increase the gas velocity dispersion ${\overline{\sigma_{\mathrm{g}}}}^{2}$ (Elmegreen et al. 1993). (2) They tend to drive gas inwards, either as a direct result of the interaction, or indirectly through the presence of tidally-induced bars (e.g., 
Noguchi 1987; Barnes \& Herquist 1996). We might expect to find starburst clumps in disturbed, gas-rich disk galaxies, such as NGC 7673.

Our study of NGC 7673 suggests it contains two classes of clumps. Clump A, the nuclear region, lies near the middle of an offset stellar bar. It is a special place, where dynamical friction and dissipation can pile up material that has been transported inwards, possibly leading to the formation of a bulge (Noguchi 1999). The other clumps are located beyond the bar. A key question is whether the outer clumps can survive for sufficiently long to carry their material inwards to make a bulge upon their disruption near the center of the galaxy, as hypothesized by Noguchi (1999).

We take Clump D, with $M_{B} \approx-16.3$ as an example. Using the Starburst99 models and a Kroupa stellar IMF (2002), we estimate that $(M / L)_{V} \approx 0.03$ for a constant SFR age of 30 Myr. Clump D then has $M \geq 10^{7} M_{\odot}$ or about $0.25 \%$ of the NGC 7673 dynamical mass; this mass is a lower limit as we have not included any gas. Taking the surface density of Clump D to be 5 times that of the mean disk, the Noguchi (1999) model then predicts the dynamical friction time scale for Clump D to reach the center of the galaxy is a few orbital periods, or $>100 \mathrm{Myr}$.

While the non-nuclear clumps have a range in ages, with Clump B being the youngest, none shows compelling evidence for star clusters with ages of $\geq 100$ Myr. This in part reflects the shallow nature of this initial survey of NGC 7673, which means we will miss any but the most massive older star clusters (see Sect. 6). However, we have seen that the integrated $V-I$ colors of the outer clumps also imply ages of $<100 \mathrm{Myr}$. Furthermore, we do not see evidence for inward migration of older clumps; both the young Clump B and older Clump D are located at similar radii. Our data therefore do not reveal the presence of long-lived clumps in NGC 7673, and so possibly, as also discussed by Noguchi (1999), this is a case where the clumps are marginally bound and less durable than in protogalaxy where more of the mass is in the form of gas.

However, we also recognize that we are observing NGC 7673 well after whatever interaction triggered its starburst. Perhaps earlier generations of clumps were more robust and already have accreted into the central region of the galaxy? After all, more than $100 \mathrm{Myr}$ are likely to have passed since the starburst was triggered, an adequate amount of time for dynamical friction to act. In this case we would expect Clump A to contain an unusually wide spread in star cluster ages from the previously dissolved clumps. Unfortunately, the interpretation of star cluster colors in the nuclear region is complicated by a combination of crowding and dust. Despite these complications, Fig. 15 shows that the redder clusters are not overly concentrated in Clump A, as might be expected if a few large clumps had dissolved in the not too distant past.

\subsection{Final fate of NGC 7673}

What will ultimately happen to NGC 7673? In their study of LBCGs, Pisano et al. (2001) calculate $\tau_{\text {gas }}$, the H II mass divided by the star formation rate, to give a simple estimate of the gas depletion timescale. Using their $\mathrm{H} \alpha$ luminosity as the SFR indicator, they find SFR $=23.5 M_{\odot} \mathrm{yr}^{-1}$ with the Kenicutt (1983) calibration, yielding $\tau_{\text {gas }}=0.18$ Gyr. If we use a more modern SFR calibration from combining a Kroupa parameterization of the IMF with Starburst99 Lyman continuum luminosity predictions, then we find SFR $\approx 12 M_{\odot} \mathrm{yr}^{-1}$ from the Pisano et al. $L(\mathrm{H} \alpha)$. Using the $L_{\mathrm{FIR}}$ from Table 1 , we get a similar result, and conclude that the current SFR is in the 10$20 M_{\odot} \mathrm{yr}^{-1}$ range. Adopting $M_{\mathrm{gas}}=1.3 M_{\mathrm{HI}}$ to allow for the presence of helium, we get an only slightly more conservative $\tau_{\text {gas }} \approx 0.5$ Gyr. This is short, and if NGC 7673 continues to form stars at the current rate, it will consume the rest of the available gas within the next $0.5 \mathrm{Gyr}$.

If it consumes all of its gas during its fast and furious starburst phase, then NGC 7673 will become a nearly gas-free disk galaxy, probably a small Sa or S0 system. If, on the other hand, the starburst is beginning its decline, this could allow a substantial fraction of the remaining $4 \times 10^{9} M_{\odot}$ of $\mathrm{H}_{\mathrm{I}}$ to form stars more sedately. The resulting system would then appear to be a late-type galaxy. $\mathrm{H}_{\mathrm{I}}$ masses of $\sim 10^{9} M_{\odot}$ are typical of small star-forming disk galaxies, and since NGC 7673 has been collisionally perturbed, we believe it would most likely have a somewhat thickened disk, such as those found in Magellanic spirals.

\section{Summary and conclusions}

NGC 7673 is a fascinatingly disturbed galaxy, with a total SFR $\left(\approx 10-20 M_{\odot} \mathrm{yr}^{-1}\right)$ and a SF intensity of for Clump B $\left(\sim 1 M_{\odot} \mathrm{yr}^{-1} \mathrm{kpc}^{-2}\right)$ that put it in the range populated by Lyman break galaxies (Pettini et al. 2001). Due to its combination of proximity for a galaxy with CNELG properties and favorable face-on orientation, it affords an excellent opportunity to explore the structure of a major starburst. While further study is needed to fully disentangle the effects of age and reddening on star cluster colors, our initial investigation reveals several interesting trends.

1. Working from WFPC 2 images, we have identified 50 star cluster candidates where we measured F255W, F555W and F814W magnitudes, and 268 with F555W and F814W photometry. While we find a broad range of star cluster colors, the bluer and brighter clusters are strongly concentrated into the main starburst "clumps".

2. In our three color sample we mainly measure clusters with ages of $<20 \mathrm{Myr}$. All of the main optical Clumps, A, B, C, $\mathrm{D}$, and $\mathrm{F}$ contain star clusters with ages of $5 \mathrm{Myr}$ or younger. However, the nuclear region, Clump A, may contain older clusters than are found in Clumps B and C. Even so we do not find an obvious age ordering of the clumps. Further observations with more filters and deeper data are needed to allow better separation of clusters which are red due to age versus young clusters reddened by interstellar dust.

3. The four brightest clumps are composed of $>50$ star clusters detected from the UV through the optical, providing $\sim 1633 \%$ of the luminosity from the clump as a whole. These results are consistent with other estimates for the fraction of light contributed by compact star clusters in starbursts (e.g., Meurer et al. 1995). 
Clump F is distinguished by not being an association of star clusters. It is instead dominated by a single object, for which we derive an age of 4-5 Myr and a mass of $3 \times 10^{5} M_{\odot}$. If the age is 4 Myr or slightly less, it should show the presence of Wolf-Rayet stars in its spectrum. Perhaps Clump F resembles a more luminous version of compact young stellar complexes with a dominant super star cluster, such as the Hodge complex in NGC 6946 (Larsen et al. 2002) or the extended knot S that is composed of luminous young stars in the Antennae (Whitmore et al. 1999).

4. The star formation process in NGC 7673 differs from that seen in normal spirals in that the disk has broken up into relatively well-defined clumps that contain most of the current starforming activity. The clumps may be understood as the result of large spatial scale instabilities in perturbed gaseous disks, as suggested by Elmegreen et al. (1993). However, it is not yet clear if the current clumps can survive long enough for dynamical friction to act to bring clumps into the center of the galaxy where they can form a bulge, as suggested by Noguchi (1999).

The unusual structure of NGC 7673 and other nearby starburst galaxies indicate that the impact of highly coeval populations of star clusters should be taken into account when considering how best to model galaxies with intense star formation. These issues extend from implications for the integrated rest-frame UV spectra to their impact on the ISM, and even to the subsequent evolution of the galactic disks (Kroupa 2002b). Furthermore, we must take into account that the formation of dense star clusters is a signature of efficient star formation, where $\geq 20 \%$ of the gas is converted into stars (e.g. Hills 1980; Lada 1999). Formation of numerous dense clusters can allow a galaxy to more efficiently convert gas into stars, thereby sustaining high SFRs in starburst systems.

Acknowledgements. This paper was improved by careful comments from the referee, Danielle Alloin. N.H. acknowledges the ESO Studentship Programme and the Wisconsin Space Grant Consortium Graduate Fellowship Program. J.S.G. and N.H. thank the University of Wisconsin Graduate School for partial support. N.H. would also like to thank Ritter Sport for many bright, chocolatey spots in many gray Garching days, and S.E. for additional encouragement and support. This project uses archival HST WFPC2 data obtained by the WFPC2 Investigation Definition Team for studies of luminous blue galaxies, and we thank the IDT for its early support of this effort.

\section{References}

Ashman, K., \& Zepf, S. 2001, AJ, 122, 1888

Babul, A., \& Rees, M. 1992, MNRAS, 255, 346

Barnes, J., \& Hernquist, L. 1996, ApJ, 471, 115

Barton, E. J., \& van Zee, L. 2001, ApJ, 550, L35

Benvenuti, P., Casini, C., \& Heidmann, J. 1982, MNRAS, 198, 825

Börngen, F., \& Kalloglian, A. 1975, ApJ, 11, 24

Calzetti, D., Kinney, A., \& Storchi-Bergmann, T. 1994, ApJ, 429, 582

Calzetti, D. 2001, PASP, 790, 1449

Casini, C., \& Heidmann, J. 1976, A\&A, 47, 371

Cerviño, M., Valls-Gabaud, D., Luridiana, V., \& Mas-Hesse, J. 2002, A\&A, 381, 51

Condon, J., \& Yin, Q. 1990, ApJ, 357, 97

Conselice, C., Bershady, M., \& Gallagher, J. 2000a, A\&A, 354, 21

Conselice, C., Gallagher, J., Calzetti, D., Homeier, N., \& Kinney, A. 2000b, AJ, 119, 79
Coupinot, G., Hecquet, J., \& Heidmann, J. 1982, MNRAS, 199, 451

Cowie, L., Hu, E., \& Songalia, A. 1995, AJ, 110, 157

de Vaucouleurs, G., de Vaucouleurs, A., \& Corwin, H. 1976, Second Reference Catalogue of Bright Galaxies (Austin: Univ. of Texas Press) (RC2)

Dettmar, R., Heidmann, J., Klein, U., \& Wielebinski, R. 1984, A\&A, 130,424

Duflot-Augarde, D., \& Alloin, D. 1982, A\&A, 112, 257

Ellis, R. S. 1997, ARA\&A, 35, 389

Elmegreen, B., Kaufman, M., \& Thomasson, M. 1993, ApJ, 412, 90

Falco, E., Kurtz, M., Geller, M., et al. 1999, PASP, 111, 438F

Ferrara, A., \& Tolstoy, E. 2000, MNRAS, 313, 291

Gallagher, J. S., \& Smith, L. J. 1999, MNRAS, 304, 540

Gallagher, J. S., Hunter, D. A., \& Bushouse, H. 1989, AJ, 97, 700

Gallagher, J. S., Conselice, C. J., Homeier, N., \& WFPC2 IDT 2000a, in Dynamics of Galaxies: from the Early Universe to the Present, ed. F. Combes, G. A. Mamon, \& V. Charmandaris, ASP Conf. Ser., 197, 309

Gallagher, J., Homeier, N., \& Conselice, C. 2000b, in Massive Stellar Clusters, ed. A. Lancon, \& C. Boily, 258

Gallego, J., Zamorano, J., Rego, M., \& Vitores, A. 1997, AJ, 475, 502

Glazebrook, K., Blake, C., Economou, F., Lilly, S., \& Colless, M. 1999, MNRAS, 306, 843

Guzmán, R., Koo, D. C., Faber, S. M., et al. 1996, ApJ, 460, L5

Guzmán, R., Koo, D. C., Phillips, A. C., et al. 1997, ApJ, 489, 559

Guzmán, R., Jangren, A., Koo, D., Bershady, M., \& Simard, L. 1998, ApJ, 495, L13

Hammer, F., Thuan, T., Cayatte, V., Guideroni, B., \& Tranh Van, T. 2000, in Building Galaxies: From the Primordial Universe to the Present, ed. F. Hammer, T. Cayatte, B. Guideroni, \& T. Tranh Van (Singapore: World Sci.)

Hernquist, L., \& Quinn, P. 1989, ApJ, 321, 1

Hernquist, L., \& Spergel, D. 1992, ApJ, 399, L117

Heyer, I. 2001, The WFPC2 Photometric CTE Monitor, WFPC2 Instrument Sci. Rep. (STScI)

Hills, J. 1980, ApJ, 225, 986

Holtzman, J. A., Hester, J. J., Casertano, S., et al. 1995a, PASP, 107, $156 \mathrm{H}$

Holtzman, J. A., Burrows, C. J., Casertano, S., et al. 1995b, PASP, 107.1065H

Homeier, N. L., \& Gallagher, J. S. 1999, ApJ, 522, 199

Howard, S., Keel, W., Byrd, G., \& Burkey, J. 1993, ApJ, 417, 502

Huchra, J. P. 1977, ApJS, 35, 171

Hunter, D. A., Gallagher, J. S., Rice, W. L., \& Gillett, F. C. 1989, ApJ, 336, 152

Hunter, D., O’Connell, R., \& Gallagher, J. 1994, AJ, 108, 84

Jangren, A., Bershady, M., Conselice, C., \& Guzán, R. 2001, ApJ, submitted

Johnson, K., Vacca, W., Leitherer, C., Conti, P., \& Lipscy, S. 1999, AJ, 117,1708

Klein, U., Heidmann, J., Wielebinski, R., \& Wunderlich, E. 1986, A\&A, 154, 373

Kennicutt, R. 1983, ApJ, 272, 54

Kinney, A., Bohlin, R., Calzetti, D., Panagia, N., \& Wyse, R. 1993, ApJS, 86, 5

Koo, D. C., Bershady, M. A., Wirth, G. D., Stanford, S. A., \& Majewski, S. R. 1994, ApJ, 427, L9

Koo, D. C., Guzmán, R., Faber, S. M., et al. 1995, ApJ, 440, L49

Kroupa, P. 2002a, Science, 295, 82

Kroupa, P. 2002b, MNRAS, 330, 707

Lada, E. 1999, in The Origin of Stars and Planetary Systems, NATO Science Ser., ed. C. Lada, \& N. Kylafis, 441 
Lançon \& Mouhcine 2000, in Stochastic Fluctuations in the Spectrophotometric Properties of Star Clusters, ASP Conf. Ser., ed. A. Lançon, \& C. Boilly, 34

Langer, N., \& Maeder, A. 1995, A\&A, 295, 685

Lanzetta, K., Yahata, N., Pascarelle, S., Chen, H.-W., \& Fernández-Soto, A. 2002, AJ, accepted

Larsen, S., Efremov, U., Elmegreen, B., et al. 2002, AJ, 567, 869

Leitherer, C., Schaerer, D., Goldader, J., et al. 1999, ApJS, 123, 3

Liu, C. T., Green, R. F., Hall, P. B., \& Osmer, P. S. 1998, AJ, 116, 1082

Mac Low, M.-M., \& Ferrara, A. 1999, ApJ, 513, 142

Mallén-Ornelas, G., Lilly, S., Crampton, D., \& Schade, D. 1999, ApJ, 518, L83

Markarian, B., \& Lipovetski, V. 1971, Astrofizika, 7, 511

Mayya, Y. 1997, ApJ, 482, 149

Meurer, G., Heckman, T., Leitherer, C., et al. 1995, AJ, 110, 2665

Miller, B., Whitmore, B., Schweizer, F., \& Fall, M. 1997, AJ, 114, 2381

Mirabel, I. F., in Star Formation in Stellar Systems. III. Canary Winter Island School of Astrophysics, ed. G. Tenorio-Tagle, M. Prieto, \& F. Sánchez (Cambridge: Cambridge U. Press), 479

Moshir, M., et al. 1990, IRAS Catalogs, The Faint Source Catalog, Version 2.0

Mulder, P., \& van Driel, W. 1996, A\&A, 309, 403

Noguchi, M. 1987, MNRAS, 228, 635
Noguchi, M. 1999, ApJ, 514, 77

Nordgren, T., Chengalur, J., Salpeter, E., \& Terzian, Y. 1997, AJ, 114, 77

Oloffson, K. 1989, A\&AS, 80, 317

Omont, A., Cox, P., Bertoldi, F., McMahon, R., Carilli, C., \& Isaak, K. 2001, A\&A, 374, 371

Origlia, L., Goldader, J., Leitherer, C., Schaerer, D., \& Oliva, E. 1998, ApJ, 514, 96

Pettini, M., Shapley, A. E., Steidel, C. C., et al. 2001, ApJ, 554, 981

Phillips, A., Guzmán, R., Gallego, J., et al. 1997, ApJ, 489, 543

Pisano, D., Kobulnicky, H., Guzmán, R., Gallego, J., \& Bershady, M. 2001, AJ, 122, 1194

Sanders, D., \& Mirabel, I. 1996, ARA\&A, 34, 749

Schlegel, D., Finkbiener, D., \& Davis, M. 1998, ApJ, 500, 525S

Schweizer, F., \& Seitzer, P. 1988, ApJ, 328, 88S

Schweizer, F., Miller, B., Whitmore, B., \& Fall, M. 1996, AJ, 112, 1839

Soifer, B. T., Boehmer, L., Neugebauer, G., \& Sanders, D. B. 1989, AJ, 98, 766S

Taniguchi, Y., \& Tamura, S. 1987, A\&A, 181, 265

Whitmore, B., \& Schweizer, F. 1995, AJ, 109, 960

Whitmore, B., Zhang, Q., Leitherer, C., et al. 1999, AJ, 118, 1551

Zackrisson, E., Bergvall, N., Olofsson, K., \& Siebert, A. 2001, A\&A, 375,814 